TITLE:

\title{
Homological and Cohomological Motives of Algebraic Varieties
}

AUTHOR(S):

masaki, hanamura

\section{CITATION:}

masaki, hanamura. Homological and Cohomological Motives of Algebraic Varieties. 代数 幾何学シンポジューム記録 1999, 1999: 191-219

ISSUE DATE:

1999

URL:

http://hdl.handle.net/2433/214696

RIGHT: 


\title{
Homological and cohomological motives of algebraic varieties
}

\author{
Masaki Hanamura
}

The framework of mixed motives in [Ha] will be shown to be suited for the study of motives of quasi-projective, possibly singular, varieties and their Chow groups. We define cohomological motives of quasi-projective varieties; they form a contravariant functor from the category of quasi-projective varieties to the triangulated category of mixed motives. There is also the functor of compactly supported cohomological motives. These functors are compatible with Weil cohomology groups, resp. compactly supported cohomology groups.

We use cubical hyperresolution (see [GNPP] for an elaborate exposition), a variant of hypercovering [De], both of which were used in the context of mixed Hodge theory. Hyperresolution has the technical advantage of being of finite length. One could formally say that this work is an adaptation of the method of hyperresolutions in the context of higher Chow groups and motives. For the hyperresolutions to exist, we assume resolutions of singularities exist for varieties over the ground field $k$. The main results of this paper are the following.

(1) (See Theorems (2.3) and (2.9).) The homological cycle complex of S. Bloch has descent property for hyperresolutions; this is a consequence of its localization property. Hence follows the existence of the contravariant functor $h_{c}$ (compactly supported cohomological motive) from. the category of quasi-projective varieties over $k$ and proper maps to the triangulated category of mixed motives $\mathcal{D}(k)$. The dual of $h_{c}$ is the "Borel-Moore homological motive" functor.

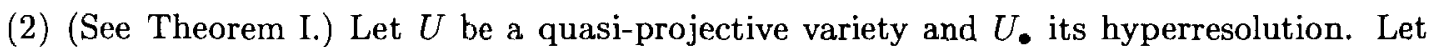
$\mathcal{Z}^{r}\left(U_{a}, \cdot\right)$ be the cycle complex of codimension of $r$ of $U_{a}$. We may form the cohomological cycle complex $\mathcal{Z}^{r}\left(U_{\bullet}\right)^{*}$ as the double complex with terms $\mathcal{Z}^{r}\left(U_{a}, \cdot\right)$; one of the differentials is the differential of each $\mathcal{Z}^{r}\left(U_{a}, \cdot\right)$, and the other differential comes from the pull-backs by the face maps of the hyperresolution. (More precisely, for the pull-backs to be defined one has to take quasi-isomorphic subcomplexes of each $\mathcal{Z}^{r}\left(U_{a}, \cdot\right)$. ) The complex is independent of the hyperresolution in the derived category, and $U \mapsto \mathcal{Z}^{r}\left(U_{\bullet}\right)^{*}$ is contravariantly functorial. The homology of this complex, denoted $\mathrm{CHC}^{r}(U, n)$, is by definition the higher Chow cohomology group, or motivic cohomology of $U$.

(3) (See Theorem II.) There exists a contravariant functor $h$ from the category of quasiprojective varieties over $k$ (and all maps) to $\mathcal{D}(k)$. The cycle complex of $h(U)$ is the cohomological cycle complex of $U$. (Each object of $\mathcal{D}(k)$ has its associated cycle complex.) $h(U)$ is the cohomological motive of $U$, and its dual $h(U)^{\vee}$ is the compactly supported homological motive of $U$.

(4) Generalizing (3), there is a contravariant functor $h$ from the category of maps of quasiprojective varieties to $\mathcal{D}(k)$, which associates to a map $f: U \rightarrow V$ its motive $h(U \stackrel{f}{\rightarrow} V)$.

(5) The motive $h(U \stackrel{f}{\rightarrow} V)$ satisfies the usual properties (distinguished triangles for triples, excision theorem).

Throughout this paper we will employ the language of mixed motives defined as diagrams of smooth projective varieties ([Ha] or $\S 4$ of this paper). This paper is a version of my preprint under the same title, which also contained the content of $[\mathrm{Ha} 1]$. In $\S 1$ we recall cubical 
hyperresolution. In $\S 2$ we review cycle complex briefly, show Theorem (2.3), and state the other main theorems. The proofs of the theorems are in $\$ \S 3,5$, and 6 .

The contravariant functor $U \mapsto C H C^{\tau}(U, 0)$ is a candidate for Chow cohomology theory $[\mathrm{Fu}]$, but falls short of satisfying all the expected properties. We will discuss this and other issues in a separate paper.

Independent of us $\mathrm{H}$. Gillet and $\mathrm{Ch}$. Soule [GS] have found that hyperresolutions - or more precisely hyperenvelopes to obtain integral results - are useful to study motives. Subsequently there appeared a related work of F. Guillen and V. Navarro-Aznar [GN]. E. Friedlander and V. Voevodsky $[\mathrm{Fr}-\mathrm{Vo}]$ also defined motivic cohomology.

Acknowledgement. We would like to thank S. Bloch, W. Fulton and Ch. Soulé for helpful advice.

We consider quasi-projective varieties over a field $k$ (namely, not necessarily irreducible, reduced quasi-projective schemes over $k$ ), or just schemes for short, and work under the assumption that desingularizatin of singularities, embedded resolution of singularities, and resolution of indeterminacy of rational maps all exist (for example char $k=0$ ). Denote the category of smooth quasi-projective (resp. quasi-projective, projective, or smooth projective) varieties by (Smooth Q-Proj/k) (resp. (Q-Proj/k), (Proj/k), or (Smooth Proj/k)).

For a map $f: X \rightarrow Y$ of varieties, the graph is the subvariety $\Gamma_{f} \subset Y \times X$ which is the image of the closed immersion $(f, i d): X \rightarrow Y \times X$.

For a cycle $z$ on $X \times Y$, its transpose ${ }^{t} z$ is the cycle $s_{*}(z)$ on $Y \times X$ where $s: X \times Y \rightarrow Y \times X$ is the isomorphism which switches the factors.

$\mathcal{D}(k)$ is the motivic category as defined in $[\mathrm{Ha}], \mathbb{Q}(r)(r \in \mathbb{Z})$ are the Tate objects. For an object $K$ of $\mathcal{D}(k)$, its dual is denoted by $K^{\vee}$, and $K(r):=K \otimes \mathbb{Q}(r)$ (Tate twists). In fact, all the constructions in this paper take place in the full subcategory $\mathcal{D}_{\text {finite }}(k)$ of motives of finite type. We recall the definition of $\mathcal{D}_{\text {finite }}(k)$ in $\S 4$.

$\mathcal{Z}^{\mathrm{a}}(K, \cdot)$ is the cycle complex of an object $K$ in $\mathcal{D}(k)$. We define

$$
\mathcal{Z}^{r}(K, \cdot)=\mathcal{Z}^{0}(K(r)[2 r], \cdot)
$$

and $\mathrm{CH}^{r}(K, n)=\mathrm{CH}^{0}(K(r)[2 r], n):=H_{n} \mathcal{Z}^{r}(K(r)[2 r], \cdot)$.

\section{$\S 1$. Cubical hyperresolutions.}

Hyperresolutions [GNPP], see also [Ca]. In the following, we denote by $I$ a finite ordered category. By definition, $I$ is a small category satisfying the following conditions:

(i) The set of objects $O b(I)$ is a finite set, and for $i, j \in O b(I), \operatorname{Hom}(i, j)$ is a finite set.

(ii) For each object $i$ of $I, \operatorname{Hom}_{I}(i, i)=\{i d\}$. If $\operatorname{Hom}_{I}(i, j)$ and $\operatorname{Hom}_{I}(j, i)$ are both nonempty, then $i=j$.

By an $I$-scheme (or a 1-diagram of schemes of type $I$ ) we mean a contravariant functor from $I$ into the category of schemes. I-schemes form a category.

For example, if $I$ is a finite ordered set, then its associated category is a finite ordered category; by definition, the associated category has $I$ as the set of objects and $\operatorname{Hom}(i, j)$ consists of a single element if $i \leq j$, and is empty if $i>j$. Another example of a finite ordered 
category we will consider is the truncated strict simplicial category $\left(\Delta_{\text {mon }}\right)_{p}$. Recall the objects of this category are $[n]=\{0,1, \cdots, n\}, n \leq p$ and the morphisms are strictly increasing maps. A $\left(\Delta_{m o n}\right)_{p}$-scheme is nothing but a truncated strict simplicial scheme.

We denote by $\square_{n}^{+}$the category associated to the partially ordered set with elements $\alpha=$ $\left(\alpha_{0}, \alpha_{1}, \alpha_{2}, \cdots, \alpha_{n}\right)$ with $\alpha_{i} \in\{0,1\} .\left(\alpha \leq \alpha^{\prime}\right.$ iff $\alpha_{i} \leq \alpha_{i}^{\prime}$ for all $i$. ) By $\square_{n}$ we mean the subset ( and subcategory) of $\square_{n}^{+}$consisting of elements (objects) except $(0,0, \cdots, 0)$. A $\square_{n}^{+}$- or $\square_{n^{-}}$ scheme is called a cubical scheme.

If $I$ and $J$ are finite ordered categories, $X$ an $I$-scheme, $Y$ a $J$-scheme, and $\phi: I \rightarrow J$ a functor, a map of 1-diagrams of schemes is a morphism of $I$-schemes $f: X \rightarrow \phi^{*} Y$. We call $\phi$ the type of $f$. Maps of 1-diagrams may be composed, and thus 1-diagrams form a category.

Let $S$ be a $I$-scheme, a 2-resolution of $S$ is a Cartesian square consisting of $I$-schemes $Z_{a b}$ $(a, b=0,1)$

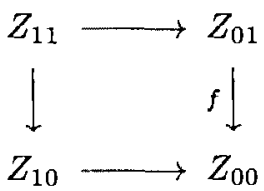

where

(1) $Z_{00}=S$,

(2) $Z_{01}$ is a smooth $I$-scheme,

(3) the horizontal arrows are closed immersions of $I$-schemes,

(4) $f$ is a proper morphism of $I$-schemes, and

(5) $Z_{10}$ contains the discriminant locus of $f$.

Let $r \geq 1$ be an integer, $X_{\bullet}^{n}$ a $\square_{n}^{+} \times I$-scheme for $1 \leq n \leq r$. Suppose that for all $n$, $1 \leq n<r$, the $\square_{n-1}^{+} \times I$-schemes $X_{00 \bullet}^{n+1}$ and $X_{1 \bullet}^{n}$ are the same. Then we define, by induction on $r$, the $\square_{r}^{+} \times I$-scheme $Z_{\bullet}=r d\left(X_{\bullet}^{1}, \cdots, X_{\bullet}^{r}\right)$ (called the reduction) as follows. If $r=1$, we define $Z_{\bullet}=X_{\bullet}^{1}$. If $r=2$, we define $Z_{\bullet \bullet}=\operatorname{rd}\left(X_{\bullet}^{1}, X_{\bullet}^{2}\right)$ by

$$
Z_{\alpha \beta}=\left\{\begin{array}{lll}
X_{0 \beta}^{1} & \text { if } & \alpha=(0,0) \\
X_{\alpha \beta}^{2} & \text { if } & \alpha \in \square_{1}
\end{array}\right.
$$

for all $\beta \in \square_{0}^{+}$, with the evident morphisms. If $r>2$, we define

$$
Z_{\bullet}=r d\left(r d\left(X_{\bullet}^{1}, \cdots X_{\bullet}^{r^{-1}}\right), X_{\bullet}^{r}\right) \text {. }
$$

(1.1) Definition. Let $S$ be an $I$-scheme. A 1-iterated cubical hyperresolution of $S$ is a $\square_{r}^{+} \times I$ scheme $Z_{\bullet}$ such that $Z_{\bullet}=r d\left(X_{\bullet}^{1}, \cdots, X_{\bullet}^{r}\right)$ where

(1) $X_{\bullet}^{1}$ is a 2-resolution of $S$,

(2) For $1 \leq n<r, X_{\bullet}^{n+1}$ is a 2-resolution of $X_{\bullet}^{n}$, and

(3) $Z_{\alpha}$ is smooth for all $\alpha \in \square_{r}$.

For an integer $n \geq 2$, define inductively the notion of $n$-iterated cubical hyperresolution of $S$ as 1-iterated cubical hyperresolution of a $(n-1)$-iterated cubical hyperresolution of $S$. Namely a $\square_{r}^{+} \times I$-scheme $X_{\bullet}$ is an $n$-iterated cubical hyperresolution if there is a sequence of 1-diagrams

$$
X_{\bullet}=X^{(n)} \rightarrow X^{(n-1)} \rightarrow \cdots \rightarrow X^{(1)} \rightarrow S
$$


of type

$$
\square_{r}^{+} \times I=\square_{r_{1}}^{+} \times \cdots \times \square_{r_{n}}^{+} \times I \rightarrow \square_{r_{1}}^{+} \times \cdots \times \square_{r_{n-1}}^{+} \times I \rightarrow \cdots \rightarrow \square_{r_{1}}^{+} \times I \rightarrow I
$$

(each map is a projection) where $X^{(i)} \rightarrow X^{(i-1)}$ is a 1-iterated cubical hyperesolution.

We will simply call an $n$-iterated cubical hyperresolution a hyperresolution of $S$. By abuse of notation, one also denotes by $X_{\bullet}$ the $\square_{r} \times I$-scheme obtained by restriction from $\square_{r}^{+}$to $\square_{r}$; this is also called hyperresolution of $S$, and often denoted with augmentation $X_{\bullet} \rightarrow S$. The number $r$ is the length of $X_{\bullet}$.

A cubical hyperresolution $X$. naturally defines a strict simplicial scheme the $n$-th term of which is

$$
X_{n}=\coprod_{|\alpha|=n+1} X_{\alpha} \quad \text { where }\left|\left(\alpha_{0}, \cdots \alpha_{m}\right)\right|=\alpha_{0}+\cdots \alpha_{m}
$$

we denote this by the same $X_{\bullet}$, the face maps by $d_{i}$ and the augmentation map by $a$.

$$
\underset{\rightarrow}{\rightarrow} X_{1} \underset{d_{1}}{\stackrel{d_{0}}{\rightrightarrows}} X_{0} \stackrel{a}{\rightarrow} S
$$

Example. Let $X$ be a quasi-projective variety, $\left\{X_{i}\right\}_{0 \leq i \leq r}$ be a finite set of smooth closed subvarieties such that $X=\cup X_{i}$. Then the $\square_{r}^{+}$-scheme defined by

$$
X_{\alpha}=\bigcap_{\alpha_{i}=1} X_{i}, \quad X_{0}=X
$$

together with the face maps induced by inclusions, is a hyperresolution of $X$, provided the $X_{\alpha}$ are all smooth. We denote this by $X_{\bullet}$. If $X$ is a simple normal crossing divisor on a smooth variety, one may take as $X_{i}$ the irreducible components of $X$, and obtain the canonical hyperresolution $X_{\bullet}$ of $X$.

It is a fact (Theorem 2.6 of [GNNP, I]) that if resolutions of singularities and embedded resolutions of singularities exist for schemes over $k$, and $I$ is a finite ordered category then any $I$-scheme $S$ over $k$ has a cubical hyperresolution. In the following we refer to cubical hyperresolution as hyperresolution.

(1.2) Definition. Let $\delta_{i}: \square_{n}^{+} \rightarrow \square_{n+1}^{+}$be the map which sends $\left(\alpha_{0}, \cdots \alpha_{n}\right)$ to $\left(\alpha_{0}, \cdots, \alpha_{i-1}, 0, \alpha_{i}, \cdots, \alpha_{n}\right)$. A face map is a map of the form

$$
\delta=\delta_{i_{p}} \circ \cdots \circ \delta_{i_{1}}: \square_{n}^{+} \rightarrow \square_{n+p}^{+}
$$

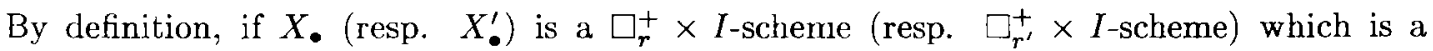
hyperresolution of an $I$-scheme $S$ (resp. $S^{\prime}$ ), a map between them is map of 1-diagrams of 
schemes of type a face map $\delta \times i d_{I}$. One then has a commutative diagram of 1-diagrams of schemes
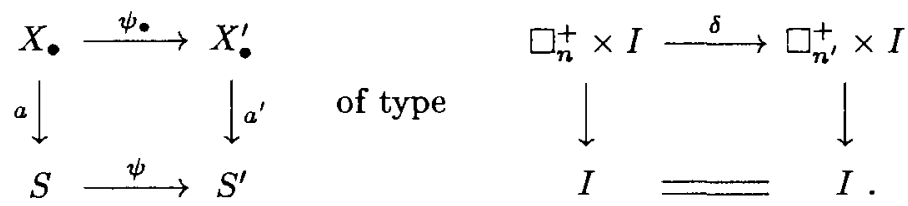

Cubical hyperresolutions of $I$-schemes form a category. A map of hyperresolutions induces the associated map of augmented strict simplicial schemes, still denoted by $\psi_{\bullet}: X_{\bullet} \rightarrow X_{\bullet}^{\prime}$.

(1.3) Maps of hyperresolutions obtained as follows are particularly useful. Let $S$ be an $I$-scheme and

$$
(\tilde{X} \rightarrow S) \rightarrow(X \rightarrow S) \rightarrow S
$$

be maps of 1-diagrams of type

$$
\square_{r}^{+} \times \square_{s}^{+} \times I \rightarrow \square_{r}^{+} \times I \rightarrow I
$$

(maps are projections) where $(X \rightarrow S)$ is an iterated hyperresolution of $S$, and $(\tilde{X} \rightarrow S)$ is an iterated hyperresolutions of $(X \rightarrow S)$. Then there are natural maps

$$
(X \rightarrow S) \stackrel{a}{\rightarrow} \tilde{X} \stackrel{b}{\leftarrow} \tilde{S}
$$

of type

$$
\square_{r}^{+} \times I \rightarrow \square_{r}^{+} \times \square_{s}^{+} \times I \leftarrow \square_{s}^{+} \times I
$$

(maps are face maps) where $\tilde{S}$ is the restriction of $\tilde{X}$ to $(0, \cdots, 0) \times \square_{s}^{+} \times I$. Note $\tilde{X}$ is of the form

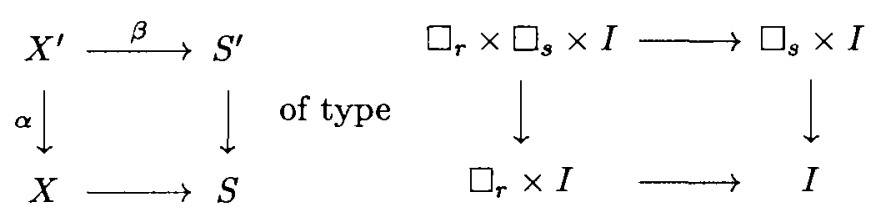

and $\tilde{S}=\left(S^{\prime} \rightarrow S\right) . \quad \tilde{S}$ is a hyperresolution of $S$. We denote the $\square_{r+s-1} \times I$-scheme $(X \leftarrow$ $\left.X^{\prime} \rightarrow S^{\prime}\right)$ by $X^{t}$.

Let $\operatorname{Hrc}(I-(Q-P r o j / k))$ denote the category of hyperresolutions of $I$-quasi-projective varieties over $k$. One has a natural functor $\operatorname{Hrc}(I-(Q-P r o j / k)) \rightarrow I-(Q-\operatorname{Proj} / k)$. Let $\Sigma$ be the class of morphisms in $\operatorname{Hrc}(I-(Q-P r o j / k))$ which induce identities on $I-(Q-P r o j / k)$, and let $\operatorname{HoHrc}(I-(Q-P r o j / k))$ be the localization of $\operatorname{Hrc}(I-(Q-P r o j / k))$ by $\Sigma$ (see [GZ] for the general notion of localization of a category). One has an induced functor

$$
\operatorname{HoHrc}(I-(Q-\operatorname{Proj} / k)) \rightarrow I-(Q-\operatorname{Proj} / k) \text {. }
$$

If $I=\underline{1}$ we write simply $\operatorname{Hrc}(Q-\operatorname{Proj} / k)$ for $\operatorname{Hrc}(1-(Q-\operatorname{Proj} / k))$. The following is [GNNP, I, Theorem 3.8]. 
(1.4) Theorem. The functor $\operatorname{HoHrc}(I-(Q-\operatorname{Proj} / k)) \rightarrow I-(Q-\operatorname{Proj} / k)$ is an equivalence of categories.

\section{§. Cycle complexes and the statements of the main theorems.}

Cycle complexes [Bl-1,2] [Bl-Le]. Let $X$ be a variety (or a scheme) over a field $k$. Define the cycle complex of $X$ as follows. Let $\square^{n}=\mathbb{A}_{k}^{n}$ with coordinates $\left(x_{1}, \cdots, x_{n}\right)$. Faces of $\square^{n}$ are intersections of codimension one faces, and the latter is of the form $\square_{i, a}^{n-1}=\left\{x_{i}=a\right\}$ where $a=0$ or 1 . A face of dimension $m$ is canonically isomorphic to $\square^{m}$. Let $\Sigma_{n}$ be the symmetric group of order $n$ acting on $\square^{n}$ by permutations of coordinates.

Let

$$
\begin{aligned}
\mathcal{Z}_{s}(X, n)= & \left\{\mathbb{Q} \text { cycle } z \text { of dimension } s+n \text { on } X \times \square^{n} \mid \text { each irreducible component of } z\right. \\
& \text { meets each face of } X \times \square^{n} \text { properly, and } z \text { is alternating with respect } \\
& \text { to the action of } \left.\Sigma_{n} .\right\}
\end{aligned}
$$

The inclusions of a codimension one faces $\delta_{i, a}: \square_{i, a}^{n-1} \hookrightarrow \square^{n}$ induce the map

$$
\partial=\sum(-1)^{i+a} \delta_{i, a}^{*}: \mathcal{Z}_{s}(X, n) \rightarrow \mathcal{Z}_{s}(X, n-1)
$$

and $\left(\mathcal{Z}_{s}(X, \cdot), \partial\right)$ is a homology complex. We call this the cycle complex (of codimension $r$ ) of $X$. By definition the (rational) higher Chow groups are the homology groups of this complex:

$$
\mathrm{CH}_{s}(X, n)=H_{n} \mathcal{Z}_{s}(X, \cdot) \text {. }
$$

For an equi-dimensional variety $X$ we set $\mathcal{Z}^{r}(X, \cdot)=\mathcal{Z}_{\operatorname{dim} X-r}(X, \cdot)$.

In $[$ Bl-Le], considering "normalized" $\mathbb{Z}$ - cycles in place of $\mathbb{Q}$-cycles, integral higher Chow group is defined, and denoted it by $\mathrm{CH}^{r}(X, n)$. In this paper we only consider the rational cycle complexes and rational Chow groups.

A proper map of varieties $f: X \rightarrow Y$ induces a map of cycle complexes $f_{*}: \mathcal{Z}_{s}(X, \cdot) \rightarrow$ $\mathcal{Z}_{s}(Y, \cdot)$. Similarly there is pull-back by flat maps.

(2.1) Localization Theorem [B]-2]. Let $X$ be a variety and $Z \subset X$ a closed subset. Then the diagram of complexes with the natural maps

$$
\mathcal{Z}_{s}(Z, \cdot) \rightarrow \mathcal{Z}_{s}(X, \cdot) \rightarrow \mathcal{Z}_{s}(X-Z, \cdot)
$$

can be extended to a distinguished triangle in the derived category.

For smooth quasi-projective varieties $V$, there is a collection of subcomplexes, called distinguished subcomplexes, that satisfy the conditions:

(i) The inclusion of a distinguished subcomplex $\mathcal{Z}^{r}(V, \cdot)^{\prime} \subset \mathcal{Z}^{r}(V, \cdot)$ is a quasi-isomorphism. If $\mathcal{Z}^{r}(V, \cdot)^{\prime}$ and $\mathcal{Z}^{r}(V, \cdot)^{\prime \prime}$ are distinguished subcomplexes of $\mathcal{Z}^{r}(V, \cdot)$, there is a third distinguished subcomplex $\mathcal{Z}^{r}(V, \cdot)^{\prime \prime \prime}$ contained in both $\mathcal{Z}^{r}(V, \cdot)^{\prime}$ and $\mathcal{Z}^{r}(V, \cdot)^{\prime \prime}$. 
(ii) If $\phi: W \rightarrow V$ is a map of smooth quasi-projective varieties, and $\mathcal{Z}^{r}(W, \cdot)^{\prime} \subset \mathcal{Z}^{r}(W, \cdot)$ is a distinguished subcomplex, there is a distinguished subcomplex $\mathcal{Z}^{r}(V, \cdot)^{\prime} \subset \mathcal{Z}^{r}(V, \cdot)$ on which $\phi^{*}: \mathcal{Z}^{r}\left(V^{\prime}, \cdot\right)^{\prime} \rightarrow \mathcal{Z}^{r}(W, \cdot)^{\prime}$ is defined.

For $V$ smooth projective, a distinguished subcomplex is one of the form $\mathcal{Z}_{W}^{r}(V, \cdot)$ where $W$ is a finite collection of subvarieties of $V$, see [Bl 1]. $\mathcal{Z}_{W}^{r}(V, n) \subset \mathcal{Z}^{r}(V, n)$ consists of cycles on $X \times \square^{n}$ that meet $W$ properly. In general take a smooth compactification $j: V \hookrightarrow \bar{V}$, a distinguished subcomplex $\mathcal{Z}^{r}(\bar{V}, \cdot)^{\prime} \subset \mathcal{Z}^{r}(\bar{V}, \cdot)$ and let $\mathcal{Z}^{r}(V, \cdot)^{\prime}$ be its image under the restriction $j^{*}: \mathcal{Z}^{r}(\vec{V}, \cdot) \rightarrow \mathcal{Z}^{r}(V, \cdot)$. This is a quasi-isomorphic subcomplex by the localization theorem. Varying $\bar{V}$ and $\mathcal{Z}^{r}(\bar{V}, \cdot)^{\prime}$, one has the collection of distinguished subcomplexes of $\mathcal{Z}^{r}(V, \cdot)$. One can verify the other properties.

Let $U$ be a quasi-projective variety, and $a: U_{\bullet} \rightarrow U$ be a hyperresolution. The double complex

$$
\stackrel{d}{\rightarrow} \mathcal{Z}_{s}\left(U_{n}, \cdot\right) \stackrel{d s}{\rightarrow} \ldots \stackrel{d s}{\rightarrow} \mathcal{Z}_{s}\left(U_{1}, \cdot\right) \stackrel{d_{s}}{\rightarrow} \mathcal{Z}_{s}\left(U_{0}, \cdot\right) \rightarrow 0
$$

(where $\mathcal{Z}_{s}\left(U_{0}, n\right)$ is placed in homological degree $n$ and $d_{*}:=\sum(-1)^{i} d_{i *}$ ) will be denoted by $\mathcal{Z}_{s}\left(U_{\bullet}\right)_{*}$. The following is a consequence of the localization theorem of cycle complexes.

(2.2) Theorem. Let

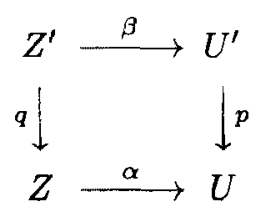

be a Cartesian square where $p$ is proper, $\beta$ is a closed immersion, and $p$ induces $p: U^{\prime}-Z^{\prime} \stackrel{\sim}{\rightarrow}$ $U-Z$. Then there is a distinguished triangle of the form

$$
\mathcal{Z}_{s}\left(Z^{\prime}\right) \stackrel{\left(\beta_{*}, q_{*}\right)}{\longrightarrow} \mathcal{Z}_{s}\left(U^{\prime}\right) \oplus \mathcal{Z}_{s}(Z) \stackrel{p \cdot-\alpha_{*}}{\longrightarrow} \mathcal{Z}_{s}(U) \stackrel{[1]}{\longrightarrow}
$$

(2.3) Theorem. The natural map of complexes $a_{*}: \mathcal{Z}_{s}\left(U_{\bullet}\right)_{*} \rightarrow \mathcal{Z}_{s}(U, \cdot)$ is a quasi- isomorphism.

Proof. One has only to prove this for 1-iterated hyperresolutions. By induction on the length $r$ of a 1-iterated hyperresolution. Let $r d\left(U_{\bullet}^{1}, \cdots, U_{\bullet}^{r}\right)$ be a hyperresolution. $U_{\bullet}^{1}$ is a 2-resolution of $U$, which is a square:

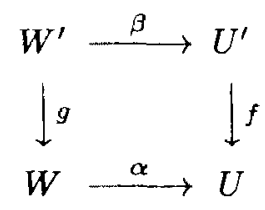

The $\square_{r-1}^{+} \times \square_{0}^{+}$-scheme $r d\left(U_{\bullet}^{2}, \cdots, U_{\bullet}^{r}\right)$ is a hyperresolution of the $\square_{0}^{+}$-scheme $g: W^{\prime} \rightarrow W$; it consists of $\square_{r-1}$-schemes $W_{\bullet}^{\prime}$ and $W_{\bullet}$, augmented to $W^{\prime}$ and $W$, respectively, and a map 
$W_{\bullet}^{\prime} \rightarrow W_{\bullet}$ augmented to $W^{\prime} \rightarrow W$.

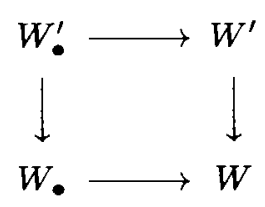

One has a map of distinguished triangles

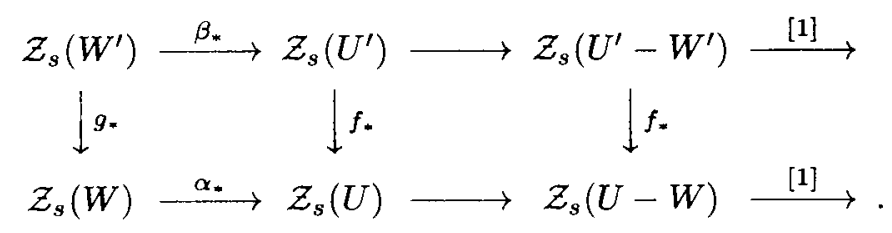

Since $U^{\prime}-W^{\prime} \stackrel{\sim}{\rightarrow} U-W$, the third horizontal arrow is a quasi-isomorphism; hence we have the distinguished triangle:

$$
\mathcal{Z}_{s}\left(W^{\prime}\right) \stackrel{\left(g_{*}, \beta_{*}\right)}{\longrightarrow} \mathcal{Z}_{s}(W) \oplus \mathcal{Z}_{s}(\tilde{U}) \stackrel{\alpha_{*}-f_{*}}{\longrightarrow} \mathcal{Z}_{s}(U) \stackrel{[1]}{\longrightarrow} .
$$

By induction hypothesis, the maps induced by the augmentations

$$
\mathcal{Z}_{s}\left(W_{\bullet}^{\prime}\right)_{*} \rightarrow \mathcal{Z}_{s}\left(W^{\prime}\right) \quad \text { and } \quad \mathcal{Z}_{s}\left(W_{\bullet}\right)_{*} \rightarrow \mathcal{Z}_{s}(W)
$$

are quasi-isomorphisms. Hence the claim for the given hyperresolution of $U$.

For a hyperresolution $U_{\bullet} \rightarrow U$, one considers the double complex

$$
\mathcal{Z}^{r}\left(U_{\bullet}\right)^{*}:=\left[\mathcal{Z}^{r}\left(U_{0}, \cdot\right)^{\prime} \stackrel{d^{*}}{\rightarrow} \mathcal{Z}^{r}\left(U_{1}, \cdot\right)^{\prime} \stackrel{d^{*}}{\rightarrow} \cdots \stackrel{d^{*}}{\rightarrow} \mathcal{Z}^{r}\left(U_{n}, \cdot\right)^{\prime} \stackrel{d^{*}}{\rightarrow} \cdots\right]
$$

where $\mathcal{Z}^{r}\left(U_{n}, \cdot\right)^{\prime}$ are distinguished subcomplexes so that the above sequence of maps $d^{*}:=$ $\sum(-i)^{i} d_{i}^{*}$ are defined. $\mathcal{Z}^{r}\left(U_{\bullet}\right)^{*}$ is well-defined up to canonical quasi-isomorphism (one is free to replace $\mathcal{Z}^{r}\left(U_{n}, \cdot\right)^{\prime}$ by smaller distinguished subcomplexes). We call it the cohomological cycle complex of $U$. If $f: U \rightarrow V$ is a map of quasi-projective varieties and $f_{\bullet}: U_{\bullet} \rightarrow V_{\bullet}$ a map of hyperresolutions over $f$, there is a map $f_{\bullet}^{*}: \mathcal{Z}^{r}\left(V_{\bullet}\right)^{*} \rightarrow \mathcal{Z}^{r}\left(U_{\bullet}\right)^{*}$, where $\mathcal{Z}^{r}\left(V_{\bullet}\right)^{*}$ is chosen so the map is defined. The cohomological cycle complex of $U$ is independent of the choice of its hyperresolution up to quasi-isomorphism as the following theorem claims.

Theorem I. Let $U$ be a quasi-projective variety, $U_{\bullet} \rightarrow U$ and $U_{\bullet}^{\prime} \rightarrow U$ be hyperresolutions of $U$, and $U_{\bullet} \rightarrow U_{\bullet}^{\prime}$ a map over $U$. Then the induced map $\mathcal{Z}^{r}\left(U_{\bullet}^{\prime}\right)^{*} \rightarrow \mathcal{Z}^{r}\left(U_{\bullet}\right)^{*}$ is a quasiisomorphism.

There is a functor

$$
\mathcal{Z}^{r}:(Q-\operatorname{Proj} / k)^{o p p} \rightarrow D(\mathbb{Q})
$$

that sends $U$ to $\mathcal{Z}^{r}\left(U_{\bullet}\right)^{*}$ and associate to a map $f: U \rightarrow V$ the induced map $\left(f_{\bullet}\right)^{*}: \mathcal{Z}^{r}\left(V_{\bullet}\right)^{*} \rightarrow$ $\mathcal{Z}^{r}\left(U_{\bullet}\right)^{*}$ where $f_{\bullet}: U_{\bullet} \rightarrow V_{\bullet}$ is a map of hyperresolutions over $f$. 
The first half together with Theorem (1.4) implies the latter half. Indeed the functor $\mathcal{Z}^{r}$ : $\operatorname{Hrc}(Q-P r o j / k)^{o p p} \rightarrow D(\mathbb{Q})$ gives rise to $\mathcal{Z}^{\tau}:$ Ho Hrc $(Q-P r o j / k)^{o p p} \rightarrow D(\mathbb{Q})$, hence the functor as stated, using the equivalence $\operatorname{HoHrc}(Q-\operatorname{Proj} / k) \rightarrow(Q-\operatorname{Proj} / k)$.

(2.4) Definition. For a quasi-projective variety $U$,

$$
\mathrm{CHC}^{r}(U, n)=H_{n} \mathcal{Z}^{r}\left(U_{\bullet}\right)^{*}
$$

this we call the higher Chow cohomology group of $U$. Higher Chow cohomology is contravariantly functorial; a map $f: U \rightarrow V$ induces a map $f^{*}: \operatorname{CHC}^{r}(V, n) \rightarrow \operatorname{CHC}^{r}(U, n)$. If $U$ is smooth, $\operatorname{CHC}^{r}(U, n)=\mathrm{CH}^{r}(U, n)$.

Write $\underline{2}$ for $\square_{0}^{+}$. A $\underline{2}$-diagram of quasi-projective varieties is nothing but a map $f: U \rightarrow V$ in $(Q-P r o j / k)$. One can consider the category of $\underline{2}$-diagrams, and denote it by $2-(Q-P r o j / k)$. For a 2-diagram $f: U \rightarrow V$, take its hyperresolution $f_{\bullet}: U_{\bullet} \rightarrow V_{\bullet}$ (more restrictive than a map of hyperresolutions over $f$, in particular $U_{\bullet}$ and $V_{\bullet}$ have the same length). Then consider the complex

$$
\text { Cone }\left[f_{\bullet}^{*}: \mathcal{Z}^{r}\left(V_{\bullet}\right)^{*} \rightarrow \mathcal{Z}^{r}\left(U_{\bullet}\right)^{*}\right]
$$

If $f^{\prime}: U^{\prime} \rightarrow V^{\prime}$ is another 2-diagram,

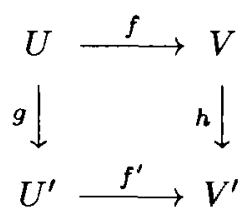

a map between them, and

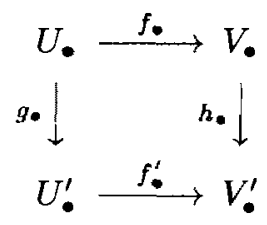

a map of hyperresolutions of the 2-diagrams, there is the induced map

$$
\left(h_{\bullet}^{*}, g_{\bullet}^{*}\right): \text { Cone } f_{\bullet}^{\prime *} \rightarrow \text { Cone } f_{\bullet}^{*} \text {. }
$$

Theorem I above can obviously be generalized to the following:

(2.5) Variant of Theorem I. Notation as above. If $(g, h)$ is the identity map of 2 -diagrams, then $\left(h_{\bullet}^{*}, g_{\bullet}^{*}\right)$ is a quasi-isomorphism. There is a functor

$$
\mathcal{Z}^{r}:\left(\underline{2}-\left(Q-P_{\text {roj }} / k\right)\right)^{\text {opp }} \rightarrow D(\mathbb{Q})
$$

that sends $f$ to Cone $\left[f_{\bullet}^{*}: \mathcal{Z}^{r}\left(V_{\bullet}\right)^{*} \rightarrow \mathcal{Z}^{r}\left(U_{\bullet}\right)^{*}\right][-1]$ and associate to $(g, h)$ the map $\left(h_{\bullet}^{*}, g_{\bullet}^{*}\right)$. 
One can thus define Chow cohomology of a 2 -diagram.

$$
\mathrm{CHC}^{r}(U \stackrel{f}{\rightarrow} V, n)=H_{n} \operatorname{Cone}\left[f_{\bullet}^{*}: \mathcal{Z}^{r}\left(V_{\bullet}\right)^{*} \rightarrow \mathcal{Z}^{r}\left(U_{\bullet}\right)^{*}\right][-1]
$$

It is contravariantly functorial. There is a long exact sequence

$$
\rightarrow \mathrm{CHC}^{r}(U \rightarrow V, n) \rightarrow \mathrm{CHC}^{r}(V, n) \stackrel{f^{*}}{\rightarrow} \mathrm{CHC}^{r}(U, n) \rightarrow \cdots .
$$

If $U \rightarrow V$ is a closed immersion, write $\mathrm{CHC}^{r}(V, U, n)$ for $\mathrm{CHC}^{r}(U \rightarrow V, n)$.

The following refines Theorem I. The category of mixed motives $\mathcal{D}(k)$ is defined in $\S 4$. There are the obvious functor $h:$ (Smooth Proj $/ k)^{o p p} \rightarrow \mathcal{D}(k)$ and the functor of cycle complexes $\mathcal{Z}^{0}: \mathcal{D}(k) \rightarrow D(\mathbb{Q})$. There is Tate twist $K(r)=K \otimes \mathbb{Q}(r)$ in $\mathcal{D}(k)$.

Theorem II. There is a functor

$$
h:(Q-P r o j / k)^{o p p} \rightarrow \mathcal{D}(k)
$$

which extends the obvious functor $h:(\text { Smooth Proj } / k)^{o p p} \rightarrow \mathcal{D}(k)$ and satisfies the following.

(i) For each $U$ in (Q-Proj/k) and its hyperresolution $U_{\bullet}$,

$$
\mathcal{Z}^{r}(h(U))=\mathcal{Z}^{0}(h(U)(r)[2 r])=\mathcal{Z}^{r}\left(U_{\bullet}\right)^{*} .
$$

(ii) If $U$ is projective,

$$
h(U)=\left[U_{0} \stackrel{\Gamma_{d}}{\longrightarrow} U_{1} \stackrel{\Gamma_{d}}{\longrightarrow} \cdots \stackrel{\Gamma_{d}}{\longrightarrow} U_{r}\right]
$$

where $\Gamma_{d}$ is the alternating sum of the graphs of the face maps.

(2.6) Variant of Theorem II. There is a functor

$$
h:(\underline{2}-(Q-\operatorname{Proj} / k))^{o p p} \rightarrow \mathcal{D}(k)
$$

which satisfies:

(i) For a 2-diagram $f: U \rightarrow V$ and its hyperresolution $f_{\bullet}: U_{\bullet} \rightarrow V_{\bullet}$,

$$
\mathcal{Z}^{r}(h(U \stackrel{f}{\rightarrow} V))=\text { Cone }\left[f_{\bullet}^{*}: \mathcal{Z}^{r}\left(V_{\bullet}\right)^{*} \rightarrow \mathcal{Z}^{r}\left(U_{\bullet}\right)^{*}\right][-1]
$$

(ii) If $U, V$ are projective then

$$
h(U \rightarrow V)=\left[\begin{array}{cccc}
U_{0} \stackrel{\Gamma_{d}}{\longrightarrow} U_{1} \stackrel{\Gamma_{d}}{\longrightarrow} \cdots \stackrel{\Gamma_{d}}{\longrightarrow} U_{r} \\
\Gamma_{f_{0}} \uparrow & \Gamma_{f_{1}} \uparrow & & \Gamma_{f_{r}} \uparrow \\
V_{0} \stackrel{\Gamma_{d}}{\longrightarrow} V_{1} \stackrel{\Gamma_{d}}{\longrightarrow} \cdots \stackrel{\Gamma_{d}}{\longrightarrow} V_{r}
\end{array}\right]
$$

where $\Gamma_{f_{i}}$ is the graph of $f_{i}$. 
(2.7) Theorem. Let

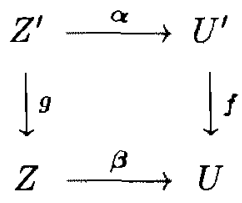

be a Cartesian square of quasi-projective varieties such that the horizontal arrows are closed immersions, and $f$ a proper map that induces $U^{\prime}-Z^{\prime} \stackrel{\sim}{\rightarrow} U-Z$. Then the pull-back

$$
(f, g)^{*}: h(U, Z) \rightarrow h\left(U^{\prime}, Z^{\prime}\right)
$$

is an isomorphism.

To state the following theorem let $\underline{3}=\{0,1,2\}$ with the usual order. An object of the category $\underline{3}-(Q-P r o j / k)$ is a diagram $U \stackrel{f}{\rightarrow} V \stackrel{g}{\rightarrow} W$ of quasi-projective varieties and maps between them. Let $D T(\mathcal{D}(k))$ be the category of distinguished triangles in $\mathcal{D}(k)$ and maps between them.

(2.8) Theorem. There is a contravariant functor

$$
(\underline{3}-(Q-P r o j / k))^{o p p} \rightarrow D T(\mathcal{D}(k))
$$

which associates to $U \stackrel{f}{\rightarrow} V \stackrel{g}{\rightarrow} W$ a distinguished triangle of the form

$$
h(V \stackrel{g}{\rightarrow} W) \rightarrow h(U \stackrel{g f}{\longrightarrow} W) \rightarrow h(U \stackrel{f}{\rightarrow} V) \stackrel{[1]}{\longrightarrow} .
$$

(2.9) Theorem. Denote by (Q-Proj/ $k$; proper) the category of quasi-projective varieties and proper maps. There exists a functor

$$
h_{c}:(\text { Q-Proj } / k ; \text { proper })^{o p p} \rightarrow \mathcal{D}(k)
$$

satisfying the properties:

(i) $h_{c}$ coincides with the functor $h$ in Theorem $I$ on the subcategory (Proj/k). If $U \hookrightarrow X$ is an open immersion into a projective variety and $Z=X-U$, there is a canonical isomorphism $h_{\mathrm{c}}(U) \stackrel{\sim}{\rightarrow} h(X, Z)$.

(ii) If $j: U^{\prime} \rightarrow U$ is an open immersion of quasi-projective varieties, there is associated a morphism $j_{*}: h_{c}\left(U^{\prime}\right) \rightarrow h_{c}(U)$. It is covariantly functorial in $j$.

(iii) if $i: Z \rightarrow U$ is a closed immersion and $j: U-Z \rightarrow U$ the open immersion of the complement, then there is a distinguished triangle

$$
h_{c}(U-Z) \stackrel{j_{*}}{\longrightarrow} h_{c}(U) \stackrel{i^{*}}{\rightarrow} h_{c}(Z) \stackrel{[1]}{\longrightarrow}
$$

functorial in $(U, Z)$. 
(iv) There is a canonical morphism $h_{c}(U) \rightarrow h(U)$ which is an isomorphism if $U$ is projective.

(v) One has

$$
\mathcal{Z}^{-s}\left(h_{c}(U)^{\vee}\right)=\mathcal{Z}_{s}(U, \cdot)
$$

(2.10) Definition. Let $U$ be a quasi-projective variety. The object $h(U)$ (resp. $h_{c}(U)$ ) is the cohomological motive (resp. it compactly supported cohomological motive) of $U$. One has

$$
\begin{array}{ll}
\mathcal{Z}^{r}(h(U))=\mathcal{Z}^{r}\left(U_{\bullet}\right)^{*} & \text { :cohomological cycle complex } \\
\mathcal{Z}^{r}\left(h_{c}(U)\right) & \text { :compactly supported cohomological cycle complex } \\
\mathcal{Z}^{-s}\left(h_{c}(U)^{\vee}\right)=\mathcal{Z}_{s}(U) & : \text { homological cycle complex } \\
\mathcal{Z}^{-s}\left(h(U)^{\vee}\right) & \text { :compactly supported homological cycle complex. }
\end{array}
$$

Taking the cohomology in degree $-n$, one has

$$
\begin{array}{ll}
C H C^{r}(U, n)=H^{-n} \mathcal{Z}^{r}(h(U)) & \text { :Chow cohomology } \\
C H C_{c p t}^{r}(U, n)=H^{-n} \mathcal{Z}^{r}(h(U)) & \text { :Chow cohomology with compact support } \\
C H_{s}(U, n)=H^{-n} \mathcal{Z}^{-s}\left(h_{c}(U)^{\vee}\right) & \text { :Chow homology } \\
C H_{s}^{c p t}(U, n)=H^{-n} \mathcal{Z}^{-s}\left(h(U)^{\vee}\right) & \text { :Chow homology with compact support. }
\end{array}
$$

Each theory is contravariantly or covariantly functorial for all maps or proper maps.

\section{§. The proof of Theorem I.}

The following is an amplification of Theorem I. If $U=U^{\prime}, f=i d$, and $Z, Z^{\prime}=\emptyset$ then one recovers Theorem I.

Theorem I' Let

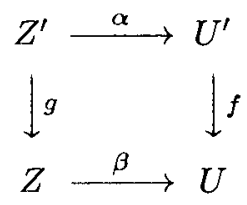

be a Cartesian square of quasi-projective varieties such that the horizontal arrows are closed immersions, and $f$ a proper map that induces $U^{\prime}-Z^{\prime} \stackrel{\sim}{\rightarrow} U-Z$. Let

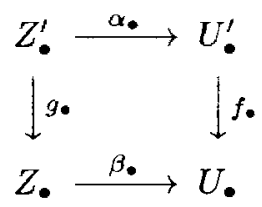


be a commutative diagram of hyperresolutions over the above square. Then the map of complexes

$$
\left(f_{\bullet}^{*}, g_{\bullet}^{*}\right): \text { Cone }\left[\beta_{\bullet}^{*}: \mathcal{Z}^{r}\left(U_{\bullet}\right)^{*} \rightarrow \mathcal{Z}^{r}\left(Z_{\bullet}\right)^{*}\right] \rightarrow \operatorname{Cone}\left[\alpha_{\bullet}^{*}: \mathcal{Z}^{r}\left(U_{\bullet}^{\prime}\right)^{*} \rightarrow \mathcal{Z}^{r}\left(Z_{\bullet}^{\prime}\right)^{*}\right]
$$

is a quasi-isomorphism.

The proof of Theorem I and Theorem I' proceeds by induction on the dimensions of the varieties involved. Let us call Theorem $\mathrm{I}_{n}$ (resp. Theorem $\mathrm{I}_{n}^{\prime}$ ) the statement of Theorem I (resp. Theorem I' ) where $\operatorname{dim} U, \operatorname{dim} \bar{U}_{\bullet}, \operatorname{dim} U_{\bullet}$ (resp. $\operatorname{dim} U_{\bullet}, \operatorname{dim} U_{\bullet}^{\prime}, \operatorname{dim} Z_{\bullet}$, and $\left.\operatorname{dim} Z_{\bullet}^{\prime}\right)$ are all $\leq n$. Here $\operatorname{dim} U_{\bullet}:=\max \operatorname{dim} U_{\alpha}$.

Proof of Theorem $\mathbf{I}_{n} \Rightarrow$ Theorem $\mathbf{I}_{n}^{\prime}$. Theorem $\mathrm{I}_{n}$ implies its variant (2.5) for 2diagrams of dimension $\leq n$. So one may take particular hyperresolutions to prove the assertion. Take a desingularization $\tilde{U} \rightarrow U^{\prime}$; let $\tilde{Z} \subset \tilde{U}$ be the inverse image of $Z$. We may take 1-iterated hyperresolutions of $Z, Z^{\prime}$, and $\tilde{Z}$ so that there is a diagram

$$
\begin{array}{ccccc}
\tilde{Z}_{\bullet} & \rightarrow & \tilde{Z} & \hookrightarrow & \tilde{U} \\
\downarrow & & \downarrow & & \downarrow \\
Z_{\bullet}^{\prime} & \rightarrow & Z^{\prime} & \hookrightarrow & U^{\prime} \\
\downarrow & & \downarrow & & \downarrow \\
Z_{\bullet} & \rightarrow & Z & \hookrightarrow & U
\end{array}
$$

Then

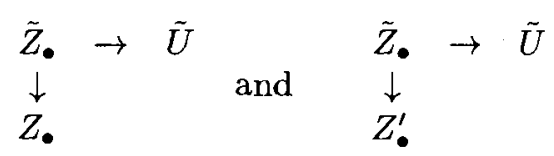

are hyperresolutions of $U, U^{\prime}$, respectively. The claim is clear in this case since both complexes are quasi-isomorphic to $\operatorname{Cone}\left[\mathcal{Z}^{r}(\tilde{U}) \rightarrow \mathcal{Z}^{r}\left(\tilde{Z}_{\bullet}\right)^{*}\right]$.

(3.1) Proposition. Assume Theorem $I_{n-1}^{\prime}$. Let

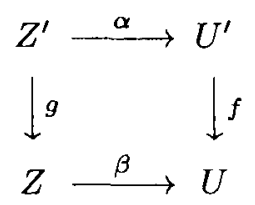

be a Cartesian square of quasi-projective varieties such that the horizontal arrows are closed immersions, $f$ a proper map that induces $U^{\prime}-Z^{\prime} \stackrel{\sim}{\rightarrow} U-Z$, and $U$ and $U^{\prime}$ are smooth. Let $g_{\bullet}: Z_{\bullet}^{\prime} \rightarrow Z_{\bullet}$ be a map of hyperresolutions over $g$. Assume the dimensions of the varieties involved (i.e. $U, U^{\prime}, Z_{\bullet}$ and $Z_{\bullet}^{\prime}$ ) are at most $n$. One has a commutative diagram

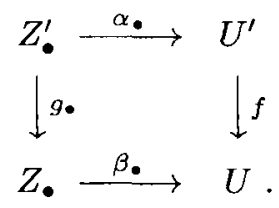


Then the map of complexes

$$
\left(f^{*}, g_{\bullet}^{*}\right): \text { Cone }\left[\beta_{\bullet}^{*}: \mathcal{Z}^{r}(U) \rightarrow \mathcal{Z}^{r}\left(Z_{\bullet}\right)^{*}\right] \rightarrow \operatorname{Cone}\left[\alpha_{\bullet}^{*}: \mathcal{Z}^{r}\left(U^{\prime}\right) \rightarrow \mathcal{Z}^{r}\left(Z_{\bullet}^{\prime}\right)^{*}\right]
$$

is a quasi-isomorphism.

Remark. It follows that if $U$ is smooth of dimension $\leq n$, and $U_{\bullet} \rightarrow U$ is a hyperresolution with $\operatorname{dim} U_{\bullet} \leq n$, then the map $\mathcal{Z}^{r}(U) \rightarrow \mathcal{Z}^{r}\left(U_{\bullet}\right)^{*}$ is a quasi-isomorphism. In fact, it is obvious for a 1-iterated hyperresolution. If $X$ is a hyperresolution of $U$, and $\tilde{X} \rightarrow X$ is a further hyperresolution, $\tilde{X}$ is of the form

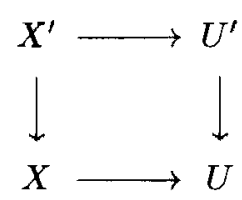

(see (1.3)). By induction on length, one knows $U^{\prime} \rightarrow U$ induces a quasi-isomorphism $\mathcal{Z}^{r}(U) \rightarrow \mathcal{Z}^{r}\left(U^{\prime}\right)^{*}$, and $X^{\prime} \rightarrow X$ also. Hence $X^{t} \rightarrow U$ also induces a quasi-isomorphism.

The following two theorems are proven in [Ha 2]. See [Fu, §6] for the case of ordinary Chow groups.

(3.2) Theorem. Let $Y$ be a smooth quasi-projective variety, $X \subset Y$ a closed smooth subvariety of codimension d. Let $f: \tilde{Y} \rightarrow Y$ be the blow-up of $Y$ along $X, \tilde{X}=f^{-1}(X)$ the exceptional divisor, $g: \tilde{X} \rightarrow X$ the induced map, and $i: X \rightarrow Y$ and $j: \tilde{X} \rightarrow \tilde{Y}$ the closed immersions.

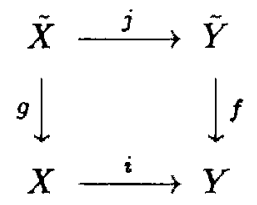

Denote by $N=N_{X} Y$ the normal bundle of $X$ in $Y$, and $E:=g^{*} N / \mathcal{O}_{N}(-1)$ the excess bundle. Then

(a) For $x \in \mathrm{CH}_{k}(X, n), f^{*} i_{*} x=j_{*}\left(c_{d-1}(E) \cdot g^{*} x\right)$.

(b) For $y \in \mathrm{CH}_{k}(Y, n), f_{*} f^{*} y=y$.

(c) If $\tilde{x} \in \mathrm{CH}_{k}(\tilde{X}, n), g_{*}(\tilde{x})=j^{*} j_{*}(\tilde{X})=0$, then $\tilde{x}=0$.

(d) There is an exact sequence

$$
0 \rightarrow \mathrm{CH}_{k}(\tilde{X}, n) \stackrel{a}{\rightarrow} \mathrm{CH}_{k}(X, n) \oplus \mathrm{CH}_{k}(\tilde{Y}, n) \stackrel{b}{\rightarrow} \mathrm{CH}_{k}(Y, n) \rightarrow 0
$$

where

$$
\begin{aligned}
a(\tilde{x}) & =\left(g_{*} \tilde{x},-j_{*} \tilde{x}\right), \\
b(x, \tilde{y}) & =i_{*}(\tilde{x})+f_{*}(\tilde{y}) .
\end{aligned}
$$

(e) If $\tilde{y} \in \mathrm{CH}_{k}(\tilde{Y}, n)$ satisfies $f_{*} \tilde{y}=j^{*} \tilde{y}=0$, then $\tilde{y}=0$. 
(f) There is an exact sequence

$$
0 \rightarrow \mathrm{CH}_{k}(X, n) \stackrel{\alpha}{\rightarrow} \mathrm{CH}_{k}(\tilde{X}, n) \oplus \mathrm{CH}_{k}(Y, n) \stackrel{\beta}{\rightarrow} \mathrm{CH}_{k}(\tilde{Y}, n) \rightarrow 0
$$

where

$$
\begin{gathered}
\alpha(x)=\left(c_{d-1}(E) \cdot g^{*} x,-i_{*}(x)\right), \\
\beta(\tilde{x}, y)=j_{*}(\tilde{x})+f^{*} y .
\end{gathered}
$$

$A$ left inverse of $\alpha$ is given by $\gamma(\tilde{x}, y)=g_{*} \tilde{x}$.

The following follows from the above.

(3.3) Theorem. Under the same hypothesis the map

$$
\left(f^{*}, g^{*}\right): \operatorname{Cone}\left[\mathcal{Z}^{r}(Y) \stackrel{i^{*}}{\rightarrow} \mathcal{Z}^{r}(X)\right] \rightarrow \operatorname{Cone}\left[\mathcal{Z}^{r}(\tilde{Y}) \stackrel{j^{*}}{\rightarrow} \mathcal{Z}^{r}(\tilde{X})\right]
$$

is a quasi-isomorphism.

Proof of Proposition (3.1). By induction on the length $r$ of $Z_{\bullet}$ (then $Z_{\bullet}^{\prime}$ has length $\leq r)$. Assume

(*) (3.1) holds if the length of $Z_{*}$ is $\leq r-1$.

By Remark to (3.1), it then follows

${ }^{* *}$ ) If $U$ is smooth of dimension $\leq n, U_{\bullet} \rightarrow U$ is a hyperresolution with $\operatorname{dim} U_{\bullet} \leq n$ and length $\leq r$, then the map $\mathcal{Z}^{r}(U) \rightarrow \mathcal{Z}^{r}\left(U_{\bullet}\right)^{*}$ is a quasi-isomorphism.

Claim 1. If $S$ is a scheme with $\operatorname{dim} S \leq n-1$ and $X \rightarrow S$ its hyperresolution of length $r$ and $\operatorname{dim} X \leq n$, then there exist open and closed $\square_{r}$-subschemes $(X)_{0},(X)_{1}$ of $X$ such that $X=(X)_{\circ} \coprod(X)_{1}$ and

(i) The subcomplex $\mathcal{Z}^{r}\left((X)_{0}\right)^{*} \subset \mathcal{Z}^{r}(X)^{*}$ is acyclic.

(ii) $\operatorname{dim}(X)_{1} \leq n-1$.

Proof. If $X$ is a 1-iterated hyperresolution, let $X$ be the reduction of a 2-resolution

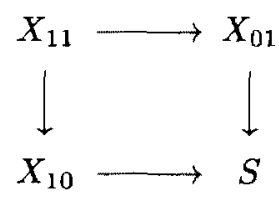

and a hyperresolution of length $r-1$

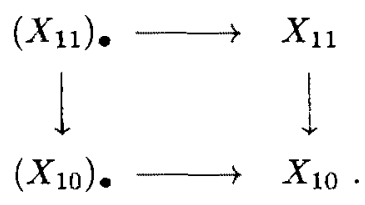


Let $\left(X_{01}\right)_{o} \subset X_{01}$ be the union of common components of $X_{11}$ and $X_{01}$. Then its complement has dimension $\leq n-1$. Let $\left(X_{11}\right)_{o}:=\left(X_{01}\right)_{o}$ and $\left[\left(X_{11}\right)_{\bullet}\right]_{o} \subset\left(X_{11}\right) \bullet$ its inverse image. Then $\left[\left(X_{11}\right)_{\bullet}\right]_{o} \rightarrow\left(X_{11}\right)_{0}$ is a hyperresolution of length $r-1$, so by $\left(^{* *}\right)$ the pull-back map

$$
\mathcal{Z}^{r}\left(\left(X_{11}\right)_{o}\right) \rightarrow \mathcal{Z}^{r}\left(\left[\left(X_{11}\right)_{\bullet}\right]_{o}\right)^{*}
$$

is a quasi-isomorphism. If one takes as $X_{o} \subset X$ the open and closed subscheme consisting of $\left(X_{10}\right)_{\bullet},\left(X_{11}\right)_{o}$ and $\left[\left(X_{11}\right)_{\bullet}\right]_{o}$, it satisfies the first condition.

Next carry out the same process on $X_{10}\left(\operatorname{resp} . X_{11}\right)$ and $\left(X_{10}\right) \bullet\left(\right.$ resp. $\left(X_{11}\right) \bullet$ in place of $X_{*}$ and $S$. Namely take $\left(X_{010}\right)_{o}$ to be the common components of $X_{010}$ and $X_{110}$, and take its inverse image. Iterate this throughout the tower of 2-resolutions. One obtains an open subscheme $X_{o} \subset X$ satisfying the two required conditions.

If $X$ is an $n$-iterated hyperresolution, one argues inductively on $n$.

Claim 2. To prove (3.1) one may assume

$\left({ }^{* *}\right) \operatorname{dim} Z_{\bullet}$ and $\operatorname{dim} Z_{\bullet}^{\prime}$ are $\leq n-1$.

Proof. Let $Z_{\circ}$ be the union of common components of $Z$ and $U$; let $\left(Z_{\bullet}\right)_{\circ} \subset Z_{\bullet}$ be the inverse image of $Z_{o}$. By $\left(^{* *}\right)$ the $\operatorname{map} \mathcal{Z}^{r}\left(Z_{o}\right) \rightarrow \mathcal{Z}^{r}\left(\left(Z_{\bullet}\right)_{0}\right)^{*}$ is a quasi-isomorphism. Similarly, if $Z_{o}^{\prime}$ be the union of common components of $Z^{\prime}$ and $U^{\prime}$, then $\mathcal{Z}^{r}\left(Z_{o}^{\prime}\right) \rightarrow \mathcal{Z}^{r}\left(\left(Z_{0}^{\prime}\right)_{o}\right)^{*}$ is a quasi-isomorphism. The assertion (3.1) will not change if we replace $\left(U, U^{\prime}, Z, Z^{\prime}\right)$ by $\left(U-Z_{o}, U^{\prime}-Z_{o}^{\prime}, Z-Z_{o}, Z^{\prime}-Z_{o}^{\prime}\right)$. Then one has $\operatorname{dim} Z, \operatorname{dim} Z^{\prime}$ are $\leq n-1$.

Apply then Claim 1 to $Z_{\bullet} \rightarrow Z$ and $Z_{\bullet}^{\prime} \rightarrow Z^{\prime}$, and replace them by open and closed subschemes with dim $\leq n-1$ without changing the quasi-isomorphism classes. The condition that $\operatorname{dim} Z_{\bullet}, \operatorname{dim} Z_{\bullet}^{\prime}$ are $\leq n-1$ is achieved.

Now assume $\left(^{*}\right)$ and $\left({ }^{* * *}\right)$. We first consider the case where $f$ is the blow-up of along a smooth center $C \subset U$ (then $C \subset Z$ ). Letting $E$ be the exceptional divisor one has a diagram

$$
\begin{array}{lllll}
E & \rightarrow & Z^{\prime} & \hookrightarrow & U^{\prime} \\
\downarrow & & \downarrow & & \downarrow \\
C & \rightarrow & Z & \hookrightarrow & U
\end{array}
$$

where all the squares are Cartesian. We apply the hypothesis Theorem $\mathrm{I}_{n-1}^{\prime}$ to the left square and Theorem (3.3) to the outside square; then we obtain the claim. Thus the claim also holds if $f$ is a succession of blow-ups along smooth centers.

In the general case, take embedded resolutions of $(U, Z)$ and $\left(U^{\prime}, Z^{\prime}\right)$, so there is a commutative diagram

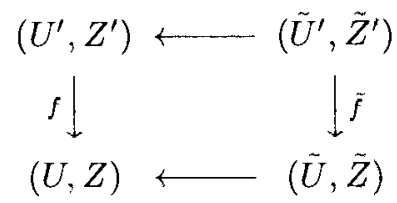

where the horizontal arrows are successions of blow-ups along smooth centers. Applying the above to the horizontal arrows, one is reduced to the following proposition.

(3.4) Proposition. Assume Theorem $I_{n-1}^{\prime}$. Let $f: X \rightarrow Y$ be a proper birational map of smooth quasi-projective varieties of dimension $n, C \subset X, D \subset Y$ be normal crossing 
divisors such that $C=\left(f^{*} D\right)_{\text {red }}$. Let

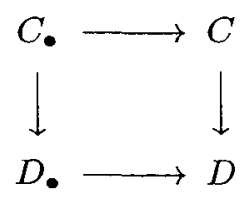

be a map of hyperresolutions. Then the map $f^{*}: \operatorname{CHC}^{r}(Y, D, m) \rightarrow \mathrm{CHC}^{r}(X, C, m)$ is injective. If moreover $f$ induces an isomorphism $X-C \stackrel{\sim}{\rightarrow} Y-D$, then $f^{*}$ is an isomorphism.

Proof. First note by $I_{n-1}^{\prime}, \operatorname{CHC}^{r}(Z, m)$ is defined for $Z$ with $\operatorname{dim} Z \leq n-1$, and contravariantly functorial. Similarly $\mathrm{CHC}^{r}(U, Z, m)$ is defined for $U$ smooth and $Z \subset U$ closed with $\operatorname{dim} Z \leq n-1$.

Since $f^{*}: \mathrm{CH}^{r}(Y, m) \rightarrow \mathrm{CH}^{r}(X, m)$ is injective, the claim is equivalent to the map

$$
f^{*}: \mathrm{CHC}^{r}(C, m) \rightarrow \mathrm{CHC}^{r}(D, m)
$$

being injective. We will prove this by induction on the number of irreducible components of $D$. Let $D_{1}$ be an irreducible component of $D, C_{1}$ a component of $C$ mapping birationally to $D_{1}, D^{\prime}=D-D_{1}$, and $C^{\prime}=\left(f^{*} D^{\prime}\right)_{\text {red }}$. One has a commutative diagram

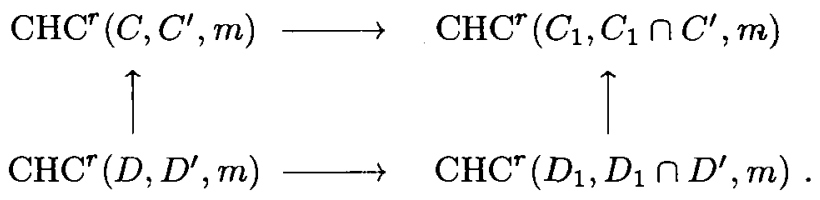

The lower arrow is an isomorphism by Theorem $I_{n-1}^{\prime}$. The right vertical map is injective by induction hypothesis on $n$. Hence the injectivity of the left vertical map. There is a map of long exact sequences

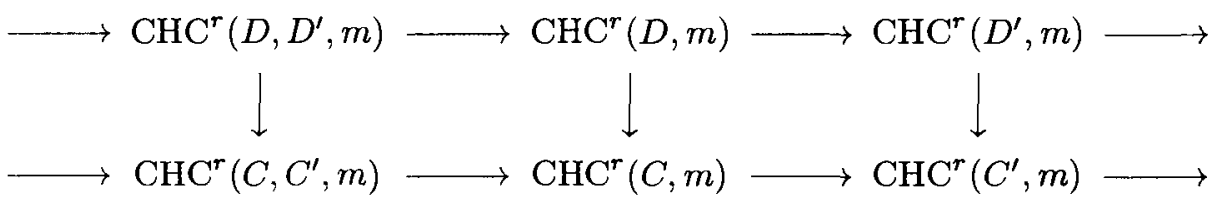

By induction hypothesis the third vertical arrow is injective. The second vertical arrow is injective by five-lemma.

Assume now $X-C \stackrel{\rightarrow}{\rightarrow} Y-D$. Take a succession of blow-ups $\tilde{f}: \tilde{Y} \rightarrow Y$ that factors through $X$, and let $\tilde{D}:=\tilde{f}^{-1}(D)_{\text {red }}$, so:

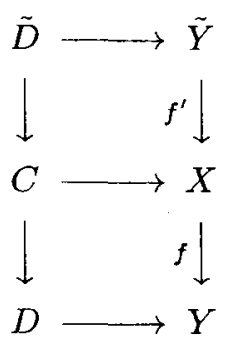


where $\tilde{f}=f \circ f^{\prime}$. Take hyperresolutions of $\tilde{D}, C$, and $D$, and one has

$$
\mathrm{CHC}^{r}(Y, D) \stackrel{f^{*}}{\longrightarrow} \mathrm{CHC}^{r}(X, C) \stackrel{f^{\prime *}}{\longrightarrow} \mathrm{CHC}^{r}(\tilde{Y}, \tilde{D}) .
$$

Since $\tilde{f}^{*}=f^{\prime *} \circ f^{*}$ is an isomorphism as in the proof of (3.1), and $f^{\prime *}$ and $f^{*}$ are injective, the latter two are both isomorphisms.

Proof of Theorem $\mathbf{I}_{n-1}^{\prime} \Rightarrow$ Theorem $\mathbf{I}_{n}$. Let $X, \tilde{X}, \tilde{S}$, etc. be as in (1.3) (with $I=\underline{1}, S=U)$. We show the maps $a, b$ both induce quasi-isomorphisms of cycle complexes. Since

$$
\mathcal{Z}^{r}\left(X^{t}\right)^{\bullet}=\operatorname{Cone}\left[\mathcal{Z}^{r}(X)^{\bullet} \oplus \mathcal{Z}^{r}\left(S^{\prime}\right)^{\bullet} \rightarrow \mathcal{Z}^{r}\left(X^{\prime}\right)^{\bullet}\right][-1]
$$

it is enough to show that both $\alpha^{*}: \mathcal{Z}^{r}(X)^{\bullet} \rightarrow \mathcal{Z}^{r}\left(X^{\prime}\right)^{\bullet}$ and $\beta^{*}: \mathcal{Z}^{r}\left(S^{\prime}\right)^{\bullet} \rightarrow \mathcal{Z}^{r}\left(X^{\prime}\right)^{\bullet}$ are quasi-isomorphisms. Assume $X$ is a $\square_{r}$-scheme, and an $n$-iterated hyperresolution of $S$. That $\alpha^{*}$ is a quasi-isomorphism follows from Remark to Proposition (3.1). To show $\beta^{*}$ is a quasi-isomorphism, one is reduced to the case $n=1$, and then to the case $r=1$. If $n=r=1, X^{\prime} \rightarrow S^{\prime}$ is a hyperresolution of the Cartesian square $X \rightarrow S$, so one concludes by Proposition (3.1).

Let $f: X \rightarrow Y$ be a map of hyperresolutions of a quasi-projective variety $S$. Consider the commutative diagram of 1-diagrams

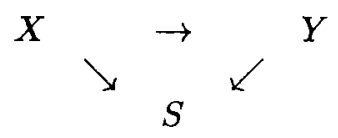

Take its total diagram, then its hyperresolution:

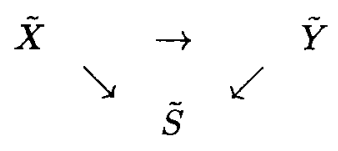

One has a commutative diagram of hyperresolutions of $S$

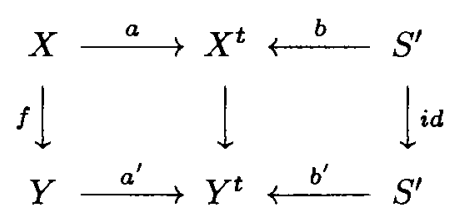

Since the four maps $a, b, a^{\prime}$ and $b^{\prime}$ induce quasi-isomorphisms of cycle complexes, so does $f$.

$\S 4$. The categories $\mathcal{D}_{\text {finite }}(k)$ and $K^{b} \mathbb{Z}(\text { Smooth } Q \text {-Proj } / k)^{o p p}$.

We collect all that is needed in this paper on the category of mixed motives. We first recall notions from [Ha, II, $\S 3$ and $\S 1]$. 
A C-complex (of abelian groups) consists of

(i) Complexes $A^{m}=\left(A^{m, \bullet}, d_{A^{m}}\right)$ (each of which is not necessarily bounded) for $m \in \mathbb{Z}$, such that for all but finitely many $m$ 's, $A^{m}=0$; and

(ii) For $m<n$, maps of graded groups

$$
F^{m, n}: A^{m, \bullet} \rightarrow A^{n, \bullet-(n-m-1)}
$$

subject to the condition

$$
F^{m, n} \circ(-1)^{m} d_{A^{m}}+(-1)^{n} d_{A^{n} \circ F^{m, n}}+\sum_{m<\ell<n} F^{\ell, n}{ }_{\circ} F^{m, \ell}=0
$$

as a map $A^{m, \bullet} \rightarrow A^{n, \bullet-n+m+2}$.

One can associate with it a complex, the total complex $\operatorname{Tot}(A)=\left(\operatorname{Tot}(A)^{\bullet}, \mathbf{d}\right)$, defined as:

$$
\operatorname{Tot}(A)^{p}=\bigoplus_{p \in \mathbb{Z}} A^{m, p-m}
$$

(which is a finite sum), and the differential $\mathbf{d}$ is the direct sum, for $m$, of $(-1)^{m} d_{A^{m}}+F^{m, n}$ : $A^{m, \bullet} \rightarrow \oplus_{n \geq m} A^{n, \bullet-(n-m-1)}$. The condition in (ii) is equivalent to $\mathrm{d}$ being a differential.

For smooth projective varieties there is another notion of distinguished subcomplexes of cycle complexes. It differs from that in $\S 2$ in that one considers only smooth projective varieties but stronger conditions, (ii), (ii) below, are satisfied. See [Ha, II, §1] for the proof.

For each smooth projective variety $X$ there is a collection of distinguished subcomplexes of $\mathcal{Z}^{r}(X, \cdot)$ satisfying:

(i) For a distinguished subcomplex $\mathcal{Z}^{r}(X, \cdot)^{\prime}$, the inclusion into $\mathcal{Z}^{r}(X, \cdot)$ is a quasi- isomorphism;

(ii) For any cycle $f \in \mathcal{Z}^{s}(X \times Y, \ell)$ and a distinguished subcomplex $\mathcal{Z}^{r+s-\operatorname{dim} X}(Y, \cdot)^{\prime}$ there is a distinguished subcomplex $\mathcal{Z}^{r}(X, \cdot)^{\prime}$ on which $f_{*}$ is defined and induces a map $f_{*}: \mathcal{Z}^{r}(X, \cdot)^{\prime} \rightarrow$ $\mathcal{Z}^{r+s-\operatorname{dim} X}(Y, \cdot+\ell)^{\prime}$

(iii) The intersection of a finite collection of distinguished subcomplexes is again distinguished.

For details on the category $\mathcal{D}(k)$ of mixed motives, we refer the reader to [Ha, II]. We will only need the subcategory $\mathcal{D}_{\text {finite }}(k)$ of mixed motives of finite type; the definitions are briefly recalled.

A finite symbol is a formal sum

$$
\bigoplus_{\alpha \in I}\left(X_{\alpha}, r_{\alpha}\right)
$$

where $X_{\alpha}$ is a smooth projective variety, $I$ a finite index set and $r_{\alpha} \in \mathbb{Z}$. We write 0 for the corresponding symbol when $I$ is an empty set.

Define dual, tensor product, and inner Hom of (a) finite symbol(s) as:

$$
\left(\oplus\left(X_{\alpha}, r_{\alpha}\right)\right)^{\vee}=\oplus\left(X_{\alpha}, \operatorname{dim} X_{\alpha}-r_{\alpha}\right)
$$




$$
\left(\oplus\left(X_{\alpha}, r_{\alpha}\right)\right) \otimes\left(\oplus\left(X_{\alpha^{\prime}}^{\prime}, r_{\alpha^{\prime}}\right)\right)=\oplus\left(X_{\alpha} \times X_{\alpha^{\prime}}^{\prime}, r_{\alpha}+r_{\alpha^{\prime}}^{\prime}\right) ;
$$

and

$$
\underline{\operatorname{Hom}}\left(\oplus\left(X_{\alpha}, r_{\alpha}\right), \oplus\left(X_{\alpha^{\prime}}^{\prime}, r_{\alpha^{\prime}}\right)\right)=\left(\oplus\left(X_{\alpha}, r_{\alpha}\right)\right)^{\vee} \otimes\left(\oplus\left(X_{\alpha^{\prime}}^{\prime}, r_{\alpha^{\prime}}\right)\right) .
$$

Define the cycle complex of a finite formal symbol by

$$
\mathcal{Z}^{0}\left(\oplus\left(X_{\alpha}, r_{\alpha}\right), \cdot\right)=\bigoplus \mathcal{Z}^{r_{\alpha}}\left(X_{\alpha}, \cdot\right)
$$

One also uses cohomological notation $\mathcal{Z}^{0}\left(\oplus\left(X_{\alpha}, r_{\alpha}\right)\right)^{-n}=\mathcal{Z}^{0}\left(\oplus\left(X_{\alpha}, r_{\alpha}\right), n\right)$.

Note there is a partially defined map

$$
\begin{gathered}
\mathcal{Z}^{0}\left(\underline{\operatorname{Hom}}\left(\left(X_{1}, r_{1}\right),\left(X_{2}, r_{2}\right)\right), \cdot\right) \otimes \mathcal{Z}^{0}\left(\underline{\operatorname{Hom}}\left(\left(X_{2}, r_{2}\right)\left(X_{3}, r_{3}\right)\right), \cdot\right) \\
--\rightarrow \mathcal{Z}^{0}\left(\underline{\operatorname{Hom}}\left(\left(X_{1}, r_{1}\right),\left(X_{3}, r_{3}\right)\right), \cdot\right)
\end{gathered}
$$

given by the composition of correspondences

$$
u \otimes v \mapsto v \circ u=p_{13 *}\left[\left(u \times X_{3}\right) \cdot\left(X_{1} \times v\right)\right] .
$$

By definition, an object of $\mathcal{D}_{f i n i t e}(k)$ is a set of data $K=\left(K^{m}\right)=\left(K^{m}, f^{m, n}\right)$ where

(i) For each integer $m, K^{m}=\oplus_{\alpha \in I(m)}\left(X_{\alpha}, r_{\alpha}\right)$, a finite symbol.

(ii) For $(m, n)$ with $m<n$, given $f^{m, n}=\left(f_{\alpha \beta}^{m, n}\right) \in \mathcal{Z}^{0}\left(\underline{\operatorname{Hom}}\left(K^{m}, K^{n}\right)\right)^{-n+m+1}$, which are subject to the conditions:

For $f^{m_{k}, m_{k+1}} \in \mathcal{Z}^{0}\left(\underline{H o m}\left(K^{m_{k}}, K^{m_{k+1}}\right)\right)^{-m_{k+1}+m_{k}+1}(k=1,2, \cdots, r)$ one has

$f^{m_{r}, m_{r+1}} \circ f^{m_{r}, m_{r-1}} \circ \cdots \circ f^{m_{1}, m_{2}}$ is defined and $\in \mathcal{Z}^{0}\left(\underline{\operatorname{Hom}}\left(K^{m_{1}}, K^{m_{r+1}}\right)\right)^{-m_{r+1}+m_{1}+r}$.

For $m<n$, one has

$$
(-1)^{n} \partial f^{m, n}+\sum_{m<\ell<n} f^{\ell, n} \circ f^{m, \ell}=0, .
$$

On the left side the compositions of the correspondences are required to be defined.

There is the functor of cycle complexes $\mathcal{Z}^{0}$ from $\mathcal{D}_{\text {finite }}(k)$ to the derived category of $\mathbb{Q}$ vector spaces. To define $\mathcal{Z}^{0}(K, \cdot)$, for each $m$ and $\alpha \in I(m)$, take a distinguished subcomplex $\mathcal{Z}^{0}\left(\left(X_{\alpha}, r_{\alpha}\right), \cdot\right)^{\prime}$ so that each $f_{\alpha, \beta}^{m, n}$ induces the map $f_{\alpha, \beta *}^{m, n}: \mathcal{Z}^{0}\left(\left(X_{\alpha}, r_{\alpha}\right), \cdot\right)^{\prime} \rightarrow \mathcal{Z}^{0}\left(\left(X_{\beta}, r_{\beta}\right), \cdot\right)^{\prime}$. We then let

$$
\mathcal{Z}^{0}\left(K^{m}, \cdot\right)^{\prime}:=\oplus_{\alpha} \mathcal{Z}^{0}\left(\left(X_{\alpha}, r_{\alpha}\right), \cdot\right)^{\prime}
$$

and have $f^{m, n}{ }_{*}: \mathcal{Z}^{0}\left(K^{m}, \cdot\right)^{\prime} \rightarrow \mathcal{Z}^{0}\left(K^{n}, \cdot+(n-m-1)\right)^{\prime}$ is defined. We define $\mathcal{Z}^{0}(K, \cdot)$ to be the total complex $\operatorname{Tot}\left(\mathcal{Z}^{0}\left(K^{m}, \cdot\right), f_{*}^{m, n}\right)$, namely the complex $(\mathbb{K}, d)$ with

$$
\mathbb{K}^{i}=\bigoplus_{j \geq i} \mathcal{Z}^{0}\left(K^{j}, j-i\right)^{\prime},
$$

and

$$
d^{i}=\sum_{j}\left((-1)^{j} \partial_{j}+\sum_{j<\ell} f_{*}^{j, \ell}\right) .
$$


Let $(K, f)$ and $(L, g)$ be objects in $\mathcal{D}_{\text {finite }}(k)$. The function cycle complex $\operatorname{Hom}(K, L)$ is defined as follows. Let $\mathcal{Z}^{0}\left(\underline{\operatorname{Hom}}\left(K^{m}, L^{m^{\prime}}\right), \cdot\right)^{\prime}$ be distinguished subcomplexes such that

For $u \in \mathcal{Z}^{0}\left(\underline{\operatorname{Hom}}\left(K^{m}, L^{m^{\prime}}\right), \cdot\right), \quad$ both $\quad u \circ f^{n, m}$ and $g^{m^{\prime}, n^{\prime}}$ ou are defined.

(This is possible since there are only finitely many non-zero $f^{n, m}$ 's and $g^{m^{\prime}, n^{\prime}}$ 's.) The cohomological complex to be defined has the group of $N$-cochains

$$
\operatorname{Hom}(K, L)^{N}=\bigoplus_{-m+m^{\prime}-p=N} \mathcal{Z}^{0}\left(\underline{\operatorname{Hom}}\left(K^{m}, L^{m^{\prime}}\right), p\right)^{\prime}
$$

The differential of this complex, which we denote by $D$, is the sum of the three kinds of maps:

$$
\begin{gathered}
(-1)^{p+m^{\prime}+n+1}\left(\circ f^{n, m}\right): \mathcal{Z}^{0}\left(\underline{H o m}\left(K^{m}, L^{m^{\prime}}\right), p\right)^{\prime} \rightarrow \mathcal{Z}^{0}\left(\underline{\operatorname{Hom}}\left(K^{n}, L^{m^{\prime}}\right), p+n^{\prime}-m^{\prime}-1\right)^{\prime}, \\
(-1)^{m^{\prime}+n^{\prime}}\left(g^{m^{\prime}, n^{\prime}} \circ\right): \mathcal{Z}^{0}\left(\underline{\operatorname{Hom}}\left(K^{m}, L^{m^{\prime}}\right), p\right)^{\prime} \rightarrow \mathcal{Z}^{0}\left(\underline{\operatorname{Hom}}\left(K^{m}, L^{n^{\prime}}\right), p+n^{\prime}-m^{\prime}-1\right)^{\prime},
\end{gathered}
$$

and

$$
(-1)^{m^{\prime}} \partial: \mathcal{Z}^{0}\left(\underline{\operatorname{Hom}}\left(K^{m}, L^{m^{\prime}}\right), \cdot\right)^{\prime} \rightarrow \mathcal{Z}^{0}\left(\underline{\operatorname{Hom}}\left(K^{m}, L^{m^{\prime}}\right), \cdot-1\right)^{\prime} .
$$

Given three objects $K, L$ and $M$, the partially defined composition map

$$
\begin{aligned}
& \operatorname{Hom}(K, L) \bullet \otimes \operatorname{Hom}(L, M)^{\bullet}--\rightarrow \operatorname{Hom}(K, M)^{\bullet} \\
& u \otimes v \mapsto v \circ u ; \quad(v \circ u)^{m, n}=\sum_{\ell \in \mathbb{Z}} v^{\ell, n} \circ u^{m, \ell}
\end{aligned}
$$

satisfies the Leibniz formula

$$
D(v \circ u)=D v \circ u+(-1)^{\operatorname{deg} v} v_{\circ} D u,
$$

where $\operatorname{deg} v$ is the total degree of $v$ in the cohomological complex. There is a quasi-isomorphic subcomplex of $\operatorname{Hom}(K, L) \bullet \otimes \operatorname{Hom}(L, M)^{\bullet}$ on which the composition is defined. See [Ha, II, $\S 1]$.

By definition

$$
\operatorname{Hom}_{\mathcal{D}_{\text {finite }}(k)}(K, L)=H^{0} \mathcal{Z}^{0}(\underline{\operatorname{Hom}}(K, L))^{\bullet} .
$$

The composition of morphisms is induced from the composition of the function complexes. A morphism $u: K \rightarrow L$ is represented by $u^{m, n} \in \operatorname{Hom}\left(K^{m}, L^{n}\right)^{-n-+m}$ (non-zero only for $m \leq n$ ) subject to the condition

$$
(-1)^{n} \partial u^{m, n}-\sum(-1)^{m+\ell} u^{\ell, n} \circ f^{m, \ell}+\sum(-1)^{\ell+n} g^{\ell, n} \circ u^{m, \ell}=0 .
$$

It defines the zero morphism if there exist $U^{m, n} \in \operatorname{Hom}\left(K^{m}, L^{n}\right)^{-n-+m-1}$ (non-zero only for $m \leq n-1)$ such that

$$
u^{m, n}=(-1)^{n} \partial U^{m, n}+\sum(-1)^{m+\ell} U^{\ell, n} \circ f^{m, \ell}+\sum(-1)^{\ell+n} g^{\ell, n} \circ U^{m, \ell} .
$$


We have the following (only the functor $h$, the duality functor and the cycle complex functor will be used in this paper), see $[\mathrm{Ha}, \mathrm{II}]$. Let $\mathbb{Q}(r)=(p t, r)[2 \tau]$, the Tate objects.

(4.1) Theorem. The category $\mathcal{D}_{\text {finite }}(k)$ has a structure of triangulated category. Moreover $\mathbb{Q}(r)$

$(1) \mathcal{D}_{\text {finite }}(k)$ has dual, tensor product, inner Hom, the unit object $\mathbb{Q}$, and the Tate objects

(2) There is a contravariant functor $h:\left(\right.$ Smooth Proj./k) $\rightarrow \mathcal{D}_{\text {finite }}(k)$.

(3) If $X$ is smooth and projective, one has

$$
\operatorname{Hom}_{\mathcal{D}_{\text {finite }}(k)}(\mathbb{Q}, h(X)(r)[2 r-m])=K_{m}(X)_{\mathbb{Q}}^{(r)}
$$

Here the right hand side is an Adams-graded piece of the $K$-group of $X$.

(4) There is the cycle complex functor $\mathcal{Z}^{0}: \mathcal{D}_{\text {finite }}(k) \rightarrow D(\mathbb{Q})$.

Let $\mathbb{Z}$ (Smooth Q-Proj $/ k)^{\text {opp }}$ be the additive category with the same objects as (Smooth Q-Proj/k) and for $V, V^{\prime}$ smooth quasi-projective

$$
\operatorname{Hom}\left(V, V^{\prime}\right)=\mathbb{Z} \operatorname{Map}\left(V^{\prime}, V\right)
$$

the free abelian group over the set of maps from $V^{\prime}$ to $V$. (This choice, rather than its opposite, will be convenient for us.) Let $K^{b} \mathbb{Z}$ (Smooth $Q$-Proj $\left./ k\right)^{o p p}$ be the homotopy category of bounded complexes in $\mathbb{Z}$ (Smooth $Q$-Proj $/ k)^{o p p}$. It is a triangulated category. An object is of the form $M=\left(M^{\bullet}, f^{\bullet}\right)$ where $M^{p}$ is smooth quasi-projective and $f^{p} \in \mathbb{Z} \operatorname{Map}\left(M^{p+1}, M^{p}\right)$. If $M, M^{\prime}$ are objects of $K^{b} \mathbb{Z}$ (Smooth $\left.Q-P r o j / k\right)^{o p p}$ one has the complex of abelian groups

$$
\operatorname{Hom}\left(M, M^{\prime}\right)^{\bullet}
$$

and $\operatorname{Hom}\left(M, M^{\prime}\right)=H^{0} \operatorname{Hom}\left(M, M^{\prime}\right)^{\bullet}$. One defines the functor of cycle complexes

$$
\mathcal{Z}^{r}: K^{b} \mathbb{Z}(\text { Smooth Q-Proj } / k)^{o p p} \rightarrow D(\mathbb{Q})
$$

as

$$
\mathcal{Z}^{r}(M)^{\bullet}=\left[\cdots \stackrel{f^{-1}}{\rightarrow} \mathcal{Z}^{r}\left(M^{0}\right)^{\bullet} \stackrel{f^{0}}{\rightarrow} \mathcal{Z}^{r}\left(M^{0}\right)^{\bullet} \stackrel{f^{1}}{\rightarrow} \cdots\right]
$$

the double complex with $\mathcal{Z}^{r}\left(M^{0}\right)^{0}$ placed in degree zero. Here we denote a distinguished subcomplex of $\mathcal{Z}^{r}\left(M^{a}\right)^{\bullet}$ by the same notation, and $f^{a}$ are the maps induced by pull-backs.

There is a natural functor

$$
\tilde{h}: \operatorname{Hrc}(Q-\operatorname{Proj} / k)^{o p p} \rightarrow K^{b} \mathbb{Z}(\text { Smooth } Q-P r o j / k)^{o p p}
$$

defines by

$$
\left(U_{\bullet} \rightarrow U\right) \mapsto\left[U_{\bullet}\right]
$$

where

$$
\left[U_{\bullet}\right]:=\left[U_{0} \stackrel{d}{\rightarrow} \cdots \stackrel{d}{\rightarrow} U_{1} \stackrel{d}{\rightarrow} U_{r}\right]
$$

( $U_{0}$ in degree 0 and $d$ is the alternating sum of the face maps). 


\section{§5. The proof of Theorem II.}

In this section we prove Theorem II and its variant (2.6), and subsequently Theorems (2.7) and (2.8).

For irreducible varieties $X \in \mathrm{Ob}$ (Smooth Proj/k) and $V \in \mathrm{Ob}$ (Smooth Q-Proj/k), let

$$
H((X, r),(V, s))^{\bullet}:=\mathcal{Z}^{\operatorname{dim} X+s-r}(X \times V, \cdot) .
$$

For $K \in \mathrm{Ob} \mathcal{D}(k)$ and $M \in \mathrm{Ob} K^{b} \mathbb{Z}(\text { Smooth } Q-P r o j / k)^{o p p}$, define the complex

$$
H(K, M)^{\bullet}:=\operatorname{Tot} H\left(K^{m}, M^{n}\right)^{\bullet}
$$

where the right hand side is the total complex, defined as in the case where both $K$ and $M$ are in $\mathrm{Ob} \mathcal{D}(k)$, where one has the function complex, see $\S 4$. Also let $H(K, M):=H^{0} H(K, M)^{\bullet}$. One has "composition of correspondences" ( $\left.M^{\prime} \in \mathrm{Ob} K^{b} \mathbb{Z}(\text { Smooth } Q-P r o j / k)^{o p p}\right)$

$$
\begin{gathered}
H(K, M)^{\bullet} \otimes \operatorname{Hom}\left(M, M^{\prime}\right)^{\bullet}--\rightarrow H\left(K, M^{\prime}\right)^{\bullet} \\
\alpha \otimes u
\end{gathered}
$$

induced from pull-back, and $\left(K^{\prime} \in \mathrm{Ob} \mathcal{D}(k)\right)$

$$
\begin{gathered}
\operatorname{Hom}\left(K^{\prime}, K\right)^{\bullet} \otimes H(K, M)^{\bullet}--\rightarrow H\left(K^{\prime}, M\right)^{\bullet} \\
v \otimes \alpha
\end{gathered}
$$

It can be shown these maps are defined on quasi-isomorphic subcomplexes by an argument similar to [Ha, II, §1]. They induce maps on 0th cohomology, e.g. $H(K, M) \otimes \operatorname{Hom}\left(M, M^{\prime}\right) \rightarrow$ $H\left(K, M^{\prime}\right)$. The composition satisfies the following associativity, both at the chain level and on cohomology.

$$
\begin{gathered}
(u \circ \alpha) \circ v=u \circ(\alpha \circ v), \\
\left(u \circ u^{\prime}\right) \circ \alpha=u \circ\left(u_{\circ}^{\prime} \alpha\right), \quad \alpha \circ\left(v \circ v^{\prime}\right)=(\alpha \circ v) \circ v^{\prime} .
\end{gathered}
$$

(5.1) Definition. Let $M \in \mathrm{Ob} K^{b} \mathbb{Z}$ (Smooth Q-Proj $\left./ k\right)^{o p p}$. A pair $(L, \alpha)$ where $L \in \mathcal{D}(k)$, $\alpha \in H(L, M)$ is a left resolution of $M$ if for any $K \in \mathcal{D}(k)$ the map

$$
\alpha \circ(-): \operatorname{Hom}_{\mathcal{D}(k)}(K, L) \rightarrow H(K, M)
$$

is an isomorphism. A left resolution is unique up to unique isomorphism.

Let $V \in$ (Smooth $Q$-Proj/k), irreducible, and take its smooth compactification, namely an open immersion $j: V \rightarrow \bar{V}$ where $\bar{V}$ is smooth projective and $D=\bar{V}-V$ is a divisor with normal crossings. Let $D^{(i)}$ be the $i$-fold intersection of the components of $D$ so one has a strict simplicial variety augmented to $\bar{V}$

$$
\cdots \underset{\rightarrow}{\rightarrow} D^{(1)} \underset{d_{1}}{\stackrel{d_{0}}{\rightarrow}} D^{(0)} \rightarrow \breve{V}
$$


Taking its associated diagram (take the alternating sum of the transposes of the graphs of the face maps) one obtains an object of $\mathcal{D}(k)$

$$
(\bar{V} \& D):=\left[\cdots \rightarrow\left(D^{(1)},-2\right) \rightarrow\left(D^{(0)},-1\right) \rightarrow \bar{V}\right]
$$

where $\bar{V}$ in degree 0 . It follows from the localization theorem that for any $X$ in (Smooth Proj/k) and any $s$,

$$
j^{*}: \mathcal{Z}_{s}(X \times(\bar{V} \& D)) \stackrel{\sim}{\rightarrow} \mathcal{Z}_{s}(X \times V) .
$$

So if we define $\alpha=\left[\Gamma_{j}\right]$ then $((\bar{V} \& D), \alpha)$ is a left resolution of $V$. By devisage there exists a left resolution for $(V, s)[i] \in \mathrm{Ob} K^{b} \mathbb{Z}$ (Smooth $\left.Q-P r o j / k\right)^{o p p}$, for any $s$ and $i$.

(5.2) Theorem. For any object $M$ of $K^{b} \mathbb{Z}(\text { Smooth } Q-P r o j / k)^{o p p}$, its left resolution $L(M) \in$ $\mathcal{D}(k)$ exists. The association $M \mapsto L(M)$ uniquely extends to a functor of triangulated categories $F: K^{b} \mathbb{Z}$ (Smooth Q-Proj $\left./ k\right)^{o p p} \rightarrow \mathcal{D}(k)$ such that the isomorphism

$$
\operatorname{Hom}_{\mathcal{D}(k)}(K, L(M)) \rightarrow H(K, M)
$$

is functorial in $M$. One has $\mathcal{Z}^{r}(M)=\mathcal{Z}^{r}(L(M))$.

Proof. The existence of left resolution is proved by induction on the "length" of an object $M$, using Proposition (5.3) below. The case $M$ is of length zero was treated above. That $M \mapsto L(M)$ extends to a functor is easy to verify using the universal property of left resolution, and the functor is triangulated by (5.3).

(5.3) Proposition. Let $M_{i}(i=1,2)$ be objects of $K^{b} \mathbb{Z}(\text { Smooth Q-Proj } / k)^{o p p},\left(L_{i}, \alpha_{i}\right)$ left

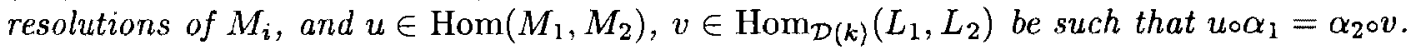

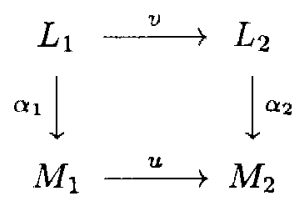

Let $M=$ Cone $u, L=$ Cone $v$. Then there is $\alpha \in H(L, M)$ such that $(L, \alpha)$ is a left resolution of $M$.

Proof. If

$$
M_{1} \rightarrow M_{2} \rightarrow M \stackrel{[1]}{\rightarrow}
$$

is a distinguished triangle, so is

$$
H\left(K, M_{1}\right)^{\bullet} \rightarrow H\left(K, M_{2}\right)^{\bullet} \rightarrow H(K, M)^{\bullet} \stackrel{[1]}{\longrightarrow} .
$$

Similarly for a distinguished triangle $K_{1} \rightarrow K_{2} \rightarrow K \stackrel{[1]}{\longrightarrow}$ one has

$$
H(K, M)^{\bullet} \rightarrow H\left(K_{2}, M\right)^{\bullet} \rightarrow H\left(K_{1}, M\right)^{\bullet} \stackrel{[1]}{\longrightarrow}
$$


distinguished. Under the assumption of the proposition, there is $\alpha \in H(L, M)$ which makes the following diagram "commutative"

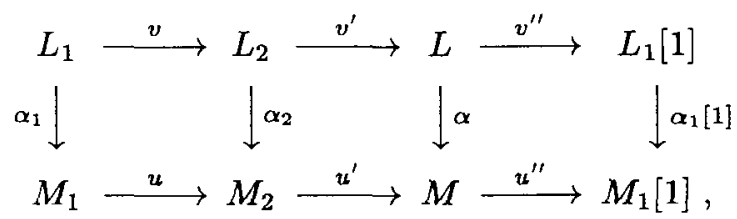

namely $\alpha \circ v^{\prime}=u^{\prime} \circ \alpha_{2}$ and $\alpha_{1}[1] \circ v^{\prime \prime}=u^{\prime \prime} \circ \alpha$. We show $(L, \alpha)$ is a left resolution of $M$. For any $K \in \mathrm{Ob} \mathcal{D}(k)$ one has a map of exact sequences

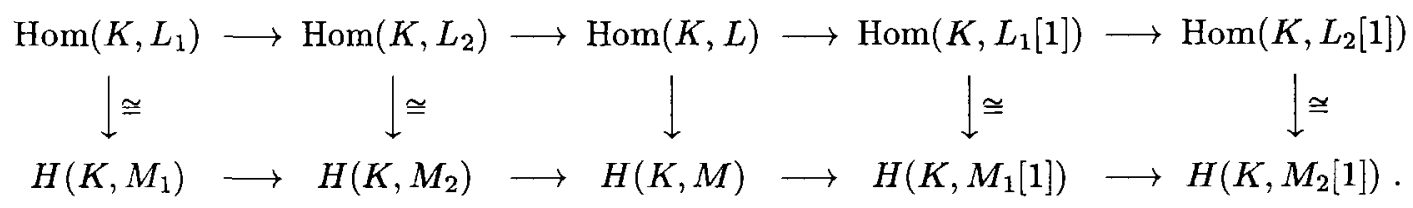

By assumption all the vertical maps except the middle one is an isomorphism; hence so is the middle one.

(5.4) Theorem. There is a unique functor

$$
h:(Q-P r o j / k)^{o p p} \rightarrow \mathcal{D}(k)
$$

such that the following square commutes (where the left vertical arrow is the natural functor).

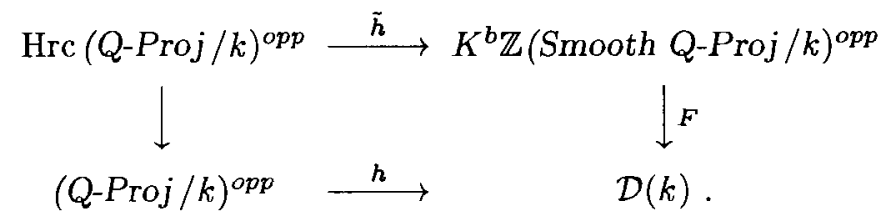

Proof. The functor $\tilde{h}_{\circ} F$ factors through Ho Hrc $(Q-P r o j / k)^{o p p}$ by Theorem I. Via the equivalence HoHrc $(Q-P r o j / k)^{o p p} \rightarrow(Q-P r o j / k)^{o p p}$ one obtains the functor $h$.

Proof of Theorem (2.6). Parallel to the proof of Theorem II, using (2.5).

Proof of Theorem (2.7). This follows from Theorem $I^{\prime}$ and the following fact.

(5.5) Proposition. Let $u: K \rightarrow L$ be a morphism in $\mathcal{D}(k)$. Suppose for any smooth projective $X$ and $s, i \in \mathbb{Z}$ the map

$$
u \circ(-): \operatorname{Hom}_{\mathcal{D}(k)}((X, s)[i], K) \rightarrow \operatorname{Hom}_{\mathcal{D}(k)}((X, s)[i], L)
$$

is an isomorphism. Then $u$ is an isomorphism. 
Proof. It follows from the hypothesis by a spectral sequence argument that for any $M$ in $\mathcal{D}(k)$, the map

$$
u_{\circ}(-): \operatorname{Hom}_{\mathcal{D}(k)}(M, K) \rightarrow \operatorname{Hom}_{\mathcal{D}(k)}(M, L)
$$

is an isomorphism. Hence the claim by Yoneda's Lemma.

Proof of Theorem (2.8). Let $U_{\bullet} \stackrel{f_{\bullet}}{\longrightarrow} V_{\bullet} \stackrel{g_{\bullet}}{\longrightarrow} W_{\bullet}$ be a hyperresolution of a $\underline{3}$-diagram $U \stackrel{f}{\rightarrow} V \stackrel{g}{\rightarrow} W$. There is a distinguished triangle in $K^{b} \mathbb{Z}(\operatorname{Smooth} Q-\operatorname{Proj} / k)$

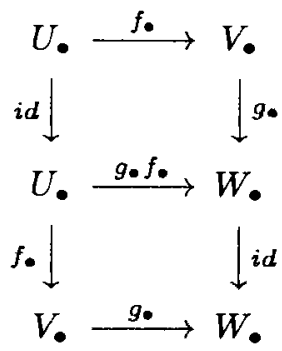

the degree one map coming from $i d: V_{\bullet} \rightarrow V_{\bullet}$. Applying the functor $F$ one has a distinguished triangle

$$
h(V \stackrel{g}{\rightarrow} W) \rightarrow h(U \stackrel{g f}{\rightarrow} W) \rightarrow h(U \stackrel{f}{\rightarrow} V) \stackrel{[1]}{\longrightarrow} .
$$

This process gives a functor

$$
\operatorname{Hrc}(\underline{3}-(Q-\operatorname{Proj} / k))^{o p p} \rightarrow D T(\mathcal{D}(k))
$$

If $\bar{U}_{\bullet} \stackrel{\bar{f}_{\bullet}}{\rightarrow} \vec{V}_{\bullet} \stackrel{\bar{g}_{\bullet}}{\rightarrow} \bar{W}_{\bullet}$ is another hyperresolution of the same $U \stackrel{f}{\rightarrow} V \stackrel{g}{\rightarrow} W$ and

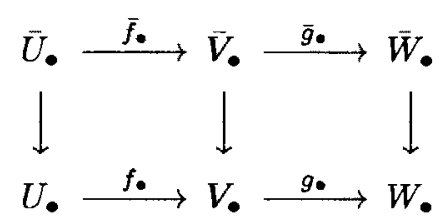

a map of hyperresolutions, the induced maps between the distinguished triangles are isomorphic, as follows from the variant of Theorem II. Thus one has the induced functor

$$
(\underline{3}-(Q-\operatorname{Proj} / k))^{o p p} \rightarrow D T(\mathcal{D}(k))
$$

\section{§. The functor $h_{c}$.}

We prove Theorem (2.9).

For $U$ in $\left(Q-P_{r o j} / k\right)$, take a projective variety $X$ and an open immersion $U \subset X$. Let $Z=X-U$ and consider the object $h(X, Z)$ in $\mathcal{D}(k)$. 
If $\left(X^{\prime}, Z^{\prime}\right)$ is another compactification of the same $U$ there is a third compactification $\left(X^{\prime \prime}, Z^{\prime \prime}\right)$ and a diagram

$$
(X, Z){ }^{f \swarrow}{ }^{\left(X^{\prime \prime}, Z^{\prime \prime}\right)} \searrow g\left(X^{\prime}, Z^{\prime}\right)
$$

and one has $f^{*}: h(X, Z) \stackrel{\sim}{\rightarrow} h\left(X^{\prime \prime}, Z^{\prime \prime}\right)$ and $g^{*}: h\left(X^{\prime}, Z^{\prime}\right) \stackrel{\sim}{\rightarrow} h\left(X^{\prime \prime}, Z^{\prime \prime}\right)$ by Theorem $(2.8)$. Therefore one obtains an isomorphism

$$
\iota:=\left(g^{*}\right)^{-1} \circ f^{*}: h(X, Z) \rightarrow h\left(X^{\prime}, Z^{\prime}\right)
$$

The isomorphism $\iota$ is independent of the choice of the diagram (*). The isomorphisms $\iota$ satisfy the cocycle condition: if $\left(X^{\prime \prime}, Z^{\prime \prime}\right)$ is a another compactification of $U$ the following diagram is commutative.

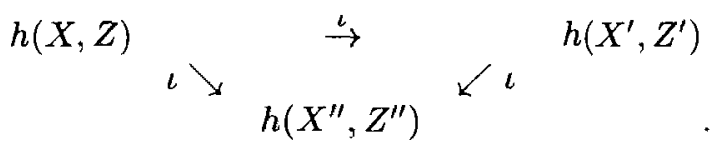

Thus define $h_{c}(U)=h(X, Z)$ up to the canonical isomorphism $\iota$. Let $f: U^{\prime} \rightarrow U$ be a proper map of quasi-projective varieties, $\left(X^{\prime}, Z^{\prime}\right),(X, Z)$ be compactifications of $U^{\prime}, U$ respectively, and $\bar{f}: X^{\prime} \rightarrow X$ be an extension of $f$. Let

$$
f^{*}: h_{c}(U) \rightarrow h_{c}\left(U^{\prime}\right)
$$

be defined by $\vec{f}^{*}: h(X, Z) \rightarrow h\left(X^{\prime}, Z^{\prime}\right)$. Since two compactifications of the same map $f$ can be dominated by a third, one shows this map is independent of the choice of $\bar{f}$. Hence one has a functor $h_{c}:(\text { Q-Proj } / k ; \text { proper })^{o p p} \rightarrow \mathcal{D}(k)$.

The composition

$$
h_{c}(U)=h(X, Z) \rightarrow h(X) \rightarrow h(U)
$$

is the canonical morphism as stated in (iv).

(ii) To show the covariant functoriality for open immersions let $j: U^{\prime} \rightarrow U$ be an open immersion. Let $U \subset X$ be an open immersion into a projective variety, $Z=X-U$, and $Z^{\prime}=X-U^{\prime}$. Then the canonical morphism

$$
h\left(X, Z^{\prime}\right) \rightarrow h(X, Z)
$$

is by definition the morphism $j_{*}: h_{c}\left(U^{\prime}\right) \rightarrow h_{c}(U)$. The independence of the choice and functoriality is proved easily.

(iii) Let $X$ be a projective variety and $A, B \subset X$ be closed subsets. One then has a distinguished triangle

$$
h(X, A \cup B) \rightarrow h(X, A) \rightarrow h(A \cup B, A) \stackrel{[1]}{\longrightarrow} .
$$

By Theorem (2.7), $h(A \cup B, A) \cong h(B, A \cap B)$. Hence follows the distinguished triangle. 
(v) If $Z \bullet \rightarrow X_{\bullet}$ is a hyperresolution of $Z \rightarrow X$,

$$
h(X, Z)^{\vee}=\left[\begin{array}{ccccccc}
Z_{0}^{\vee} & \stackrel{\Gamma_{d}}{\longleftarrow} Z_{1}^{\vee} & \Gamma_{d} & \cdots & \Gamma_{d} & Z_{r}^{\vee} \\
\downarrow & & \downarrow & & & \downarrow \\
X_{0}^{\vee} & \longleftarrow & \Gamma_{1}^{\vee} & \longleftarrow & & & \\
\Gamma_{d} & \cdots & \Gamma_{d} & X_{r}^{\vee}
\end{array}\right]
$$

where $X_{0}^{\vee}$ is placed in degree 0 . Since $\mathcal{Z}^{-s}\left(X^{\vee}\right)=\mathcal{Z}_{s}(X)$ for $X$ smooth projective, one has

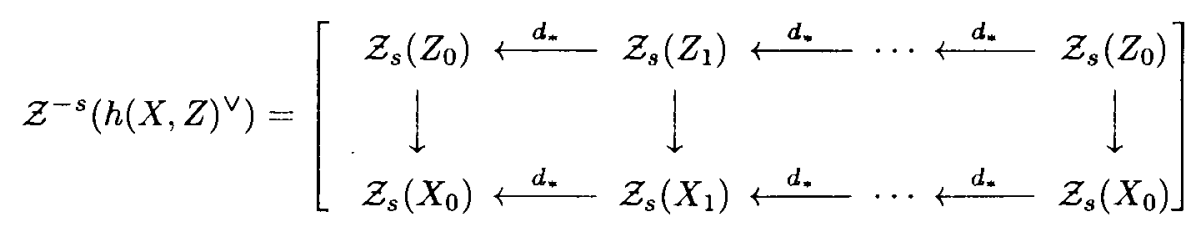

the maps are push-forwards by the face maps of $Z_{\bullet}, X_{\bullet}$ and the maps $Z_{\bullet} \rightarrow X_{\bullet}$. By Theorem (2.3) the natural map

$$
\mathcal{Z}^{-s}\left(h(X, Z)^{\vee}\right) \rightarrow \operatorname{Cone}\left[\mathcal{Z}_{s}(Z) \rightarrow \mathcal{Z}_{s}(X)\right]
$$

is a quasi-isomorphism, and by Theorem (2.1) there is a natural quasi-isomorphism

$$
\operatorname{Cone}\left[\mathcal{Z}_{s}(Z) \rightarrow \mathcal{Z}_{s}(X)\right] \rightarrow \mathcal{Z}_{s}(U) .
$$

Hence one has a quasi-isomorphism $\mathcal{Z}^{-s}\left(h(X, Z)^{\vee}\right) \rightarrow \mathcal{Z}_{s}(U)$. This quasi-isomorphism is compatible with different choices of $(X, Z)$.

Remark. To show (2.9) one only needs a functor

$$
h:(\underline{2}-(\operatorname{Proj} / k))^{o p p} \rightarrow \mathcal{D}(k)
$$

the restriction of $h$ in Theorem (2.6) to the subcategory of 2-diagrams of projective varieties. The existence of such a functor is also proved in [Ha 1].

\section{References.}

[Bl-1] Bloch, S. : Algebraic cycles and higher K-theory, Adv. Math. 61 (1986), 267-304.

[Bl-2] Bloch, S. : The moving lemma for higher Chow groups, J. Alg. Geom. (1994).

[Bl-Le] Bloch, S. and Levine, M. : Book in preparation.

[Ca] Carlson, J. : Polyhedral resolutions of algebraic varieties, Trans. Amer. Math. Soc. $292(1985) 595-612$.

[De] Deligne, P. : Theorie de Hodge II, Publ. Math. IHES 40 (1972), 5-57; III, Publ. Math. IHES 44 (1975), 6-77.

[Fr-Vo] Friedlander, E. and Voevodsky, V. : Bivariant cycle cohomology, preprint.

[Fu] Fulton, W. : Intersection Theory, Springer-Verlag, 1984. 
[GZ] Gabriel, P., Zisman M. : Calculus of Fractions and Homotopy Theory, Springer-Verlag, 1967.

[GS] Gillet, H., Soulé, Ch. : Descent, motives and $K$-theory, J, reine. angew. Math. 478 (1996), 127-176.

[GNPP] Guillen, F., Navarro-Aznar, V., Pascual-Gainza, P., and Puerta, F. : Hyperresolutions cubiques et descente cohomologique, LNM 1335, Springer-Verlag, 1988.

[GN] Guillen, F., Navarro-Aznar, V.: Un critére d'extension d'un foncteur défini sur les schémas lisses, preprint.

[Ha ] Hanamura, M. : Mixed motives and algebraic cycles I, Math. Res. Letters 2 (1995), 811-821; II, III, preprint.

[Ha 1] Hanamura, M. : The mixed motive of a projective variety, preprint.

[Ha 2] Hanamura, M. : Blow-ups and mixed motives, in preparation.

[Le] Levine, M. : Mixed Motives, Mathematical Surveys and Monographs, vol. 57, 1998, American Math. Soc.

[Li] Lichtenbaum, S. : Suslin homology and Deligne 1-motives, preprint.

Graduate School of Mathematics

Kyushu University

Hakosaki, Fukuoka, 812-8581, Japan 